ANALYSIS \& PDE

Volume $3 \quad$ No. $2 \quad 2010$

\author{
GREG KNESE
}

\title{
POLYNOMIALS WITH NO ZEROS ON THE BIDISK
}




\title{
POLYNOMIALS WITH NO ZEROS ON THE BIDISK
}

\author{
GREG KNESE
}

We prove a detailed sums of squares formula for two-variable polynomials with no zeros on the bidisk $\mathbb{D}^{2}$, extending previous such formulas by Cole and Wermer and by Geronimo and Woerdeman. Our formula is related to the Christoffel-Darboux formula for orthogonal polynomials on the unit circle, but the extension to two variables involves issues of uniqueness in the formula and the study of ideals of twovariable orthogonal polynomials with respect to a positive Borel measure on the torus which may have infinite mass. We present applications to two-variable Fejér-Riesz factorizations, analytic extension theorems for a class of bordered curves called distinguished varieties, and Pick interpolation on the bidisk.

1. Introduction 109

2. An example 114

3. Sums of squares and uniqueness 114

$\begin{array}{ll}\text { 4. Preliminaries } & 117\end{array}$

5. General properties of orthogonal polynomials on $\mathbb{T}^{2} \quad 121$

6. OC measures 124

7. Bernstein-Szegő measures 130

8. Proof of the main theorem 135

9. Polynomials with unique decompositions 135

10. Application: Fejér-Riesz factorization $\quad 138$

11. Application: distinguished varieties 143

12. Application: Agler's Pick interpolation theorem 146

$\begin{array}{ll}\text { 13. Questions } & 147\end{array}$

$\begin{array}{ll}\text { Notational index and conventions } & 148\end{array}$

$\begin{array}{lr}\text { Acknowledgements } & 148\end{array}$

$\begin{array}{ll}\text { References } & 148\end{array}$

\section{Introduction}

Let $q \in \mathbb{C}[z, w]$ be a polynomial of degree $(n, m)$ (degree $n$ in $z$ and degree $m$ in $w$ ). Suppose $q$ has no zeros on the unit bidisk $\mathbb{D}^{2}:=\mathbb{D} \times \mathbb{D} \subset \mathbb{C}^{2}$. Then, $q$ satisfies the following "sums of (Hermitian) squares" formula: there exist polynomials $A_{j} \in \mathbb{C}[z, w]$, for $j=1, \ldots, n$, and $B_{k} \in \mathbb{C}[z, w]$, for $k=1, \ldots, m$ such that

$$
|q(z, w)|^{2}-|\overleftarrow{q}(z, w)|^{2}=\left(1-|z|^{2}\right) \sum_{j=1}^{n}\left|A_{j}(z, w)\right|^{2}+\left(1-|w|^{2}\right) \sum_{k=1}^{m}\left|B_{k}(z, w)\right|^{2}
$$

MSC2000: primary 42C05; secondary 47A57, 46C07, 42B05, 14M12.

Keywords: bidisk, Christoffel-Darboux, sums of squares, Fejér-Riesz, orthogonal polynomials, distinguished varieties, Pick interpolation, Andô's inequality, Bernstein-Szegő measures, torus, stable polynomials. 
where $\overleftarrow{q}$ is the "reflection" of $q$ :

$$
\overleftarrow{q}(z, w)=z^{n} w^{m} \overline{q\left(\frac{1}{\bar{z}}, \frac{1}{\bar{w}}\right)} .
$$

This was first proved in [Cole and Wermer 1999]. Here is an example.

Example 1.1. The polynomial $q(z, w)=2-z-w$ has degree $(1,1)$ and no zeros on $\mathbb{D}^{2}$. The reflection of $q$ is $\overleftarrow{q}(z, w)=2 z w-w-z$. The sum of squares decomposition for $q$ is rather simple:

$$
|2-z-w|^{2}-|2 z w-w-z|^{2}=\left(1-|z|^{2}\right) 2|1-w|^{2}+\left(1-|w|^{2}\right) 2|1-z|^{2} .
$$

There are several reasons why we deem the Cole-Wermer formula interesting. First, it can be used to give direct proofs of Andô's inequality from operator theory (in [Cole and Wermer 1999]) and Agler's Pick interpolation theorem for the bidisk (see Section 12 for this simple derivation). Second, (1-1) can be thought of as a two-variable version of the Christoffel-Darboux formula for orthogonal polynomials on the unit circle. The Christoffel-Darboux formula is fundamental in the theory of orthogonal polynomials on the unit circle [Simon 2005; 2008]. Third, the most obvious analogue of (1-1) in three or more variables is false as it would imply a three operator version of Andô's inequality (something known to be false). Fourth, (1-1) can be used to prove a determinantal representation for a class of algebraic curves in $\mathbb{C}^{2}$ called distinguished varieties (as in [Knese 2009]).

One drawback to the Cole-Wermer formula is that the sums of squares decomposition is not unique.

\section{Example 1.2.}

$$
\begin{aligned}
|3-z-w|^{2}-|3 z w-z-w|^{2} & \\
= & \left(1-|z|^{2}\right) 3\left|\frac{1-\sqrt{5}}{2}+\frac{1+\sqrt{5}}{2} w\right|^{2}+\left(1-|w|^{2}\right) 3\left|\frac{1+\sqrt{5}}{2}+\frac{1-\sqrt{5}}{2} z\right|^{2} \\
& =\left(1-|z|^{2}\right) 3\left|\frac{1+\sqrt{5}}{2}+\frac{1-\sqrt{5}}{2} w\right|^{2}+\left(1-|w|^{2}\right) 3\left|\frac{1-\sqrt{5}}{2}+\frac{1+\sqrt{5}}{2} z\right|^{2}
\end{aligned}
$$

(Example 2.1 below is more interesting.) It turns out that we can make the Cole-Wermer sums of squares decomposition unique if we require more.

Here is an abridged version of our main theorem. We will fill in more details in Theorem 8.1. All new terminology in the theorem is explained immediately following its statement.

Theorem 1.3. Let $q \in \mathbb{C}[z, w]$ be almost stable and $\operatorname{deg} q \leq(n, m)$. Then, there exist vector polynomials $\mathbf{E} \in \mathbb{C}^{n}[z, w]$ and $\mathbf{F} \in \mathbb{C}^{m}[z, w], \operatorname{deg} \mathbf{E} \leq(n-1, m), \operatorname{deg} \mathbf{F} \leq(n, m-1)$ such that

(1) $\mathbf{E}$ is horizontally $\mathbb{D}$-stable;

(2) $\mathbf{F}$ is vertically $\mathbb{E}$-stable;

(3) the following formula holds

$$
|q(z, w)|^{2}-|\overleftarrow{q}(z, w)|^{2}=\left(1-|z|^{2}\right)|\mathbf{E}(z, w)|^{2}+\left(1-|w|^{2}\right)|\mathbf{F}(z, w)|^{2} ;
$$

(4) $\mathbf{E} \in \mathbb{C}^{n}[z, w]$ and $\mathbf{F} \in \mathbb{C}^{m}[z, w]$ satisfying items (1) and (3) are unique up to unitary multiplication. 
Definition 1.4. A polynomial is $p \in \mathbb{C}[z, w]$ is stable if $p$ has no zeros on $\overline{\mathbb{D}^{2}}$. A polynomial $p \in \mathbb{C}[z, w]$ is almost stable if $p$ has no zeros on $\mathbb{D}^{2}$ and finitely many zeros on $\mathbb{T}^{2}$.

For instance, $p(z, w)=3-z-w$ is stable; $q(z, w)=2-z-w$ is almost stable.

Notation 1.5. We use $\mathbb{T}$ to denote the unit circle $\partial \mathbb{D}$ and $\mathbb{T}^{2}$ is the two-dimensional torus, or just torus; $\mathbb{E}:=\mathbb{C} \backslash \overline{\mathbb{D}}$ is the exterior disk. We use $\mathbb{C}^{N}[z]$ to denote the set of $\mathbb{C}^{N}$-valued polynomials in the variable $z$; likewise, we use $\mathbb{C}^{N}[z, w]$ to denote the set of $\mathbb{C}^{N}$-valued polynomials in $z$ and $w$. We define

$$
\Lambda_{N}(z):=\left(\begin{array}{c}
1 \\
z \\
\vdots \\
z^{N-1}
\end{array}\right) \in \mathbb{C}^{N}[z] .
$$

If $\mathbf{E}(z, w)=\sum_{j=0}^{n-1} \mathbf{E}_{j}(w) z^{j} \in \mathbb{C}^{N}[z, w]$ has degree less than $n$ in $z$, we will frequently write $\mathbf{E}$ in the matrix form

$$
\mathbf{E}(z, w)=\left(\mathbf{E}_{0}(w), \mathbf{E}_{1}(w), \ldots, \mathbf{E}_{n-1}(w)\right) \Lambda_{n}(z)=E(w) \Lambda_{n}(z),
$$

where $E(w)=\left(\mathbf{E}_{0}(w), \mathbf{E}_{1}(w), \ldots, \mathbf{E}_{n-1}(w)\right)$ is an $N \times n$ matrix valued polynomial in $w$.

Similarly, if $\mathbf{F} \in \mathbb{C}^{M}[z, w]$ has degree less than $m$ in $w$, we may write

$$
\mathbf{F}(z, w)=F(z) \Lambda_{m}(w),
$$

where $F(z)$ is an $M \times m$ matrix polynomial in $z$.

Definition 1.6. Let $\Omega \subset \mathbb{C}$. Under the conventions above, we say $\mathbf{E}$ is horizontally $\Omega$-stable if $E(w)$ has full rank for all $w \in \Omega$; we say $\mathbf{F}$ is vertically $\Omega$-stable if $F(z)$ has full rank for all $z \in \Omega$.

Typically, $\Omega$ is one of following sets: $\mathbb{D}, \mathbb{E}, \mathbb{D} \cup \mathbb{E}$, or $\mathbb{D}$ unioned with a subset of $\mathbb{T}$.

Let us explain the terminology. For fixed $w_{0} \in \mathbb{D}$, call the set $\left\{\left(z, w_{0}\right): z \in \mathbb{C}\right\}$ a horizontal line over $\mathbb{D}$. Supposing $N \leq n$, being horizontally $\mathbb{D}$-stable is equivalent to saying the image of

$$
\mathbf{E}: \mathbb{C}^{2} \rightarrow \mathbb{C}^{N}
$$

when restricted to a horizontal line over $\mathbb{D}$ sits in no linear subspace of dimension less than $N$. The reason is simple:

$$
\mathbf{E}\left(z, w_{0}\right)=E\left(w_{0}\right) \Lambda_{n}(z),
$$

and when $E\left(w_{0}\right)$ has full rank, the span of the right hand side as $z$ varies over $\mathbb{C}$ is $\mathbb{C}^{N}$. Being horizontally $\mathbb{D}$-stable is much stronger than saying $\mathbf{E}$ is nonvanishing on $\mathbb{C} \times \mathbb{D}$. A similar interpretation holds for $\mathbf{F}$ and "vertical" objects.

Notation 1.7. We let $|\cdot|$ denote the standard norm on $\mathbb{C}^{N}$ (where the $N$ will be understood from context) and therefore if $\mathbf{E}=\left(e_{1}, \ldots, e_{N}\right)^{t} \in \mathbb{C}^{N}[z, w]$, then

$$
|\mathbf{E}(z, w)|^{2}=\sum_{j=1}^{N}\left|e_{j}(z, w)\right|^{2}
$$

is evaluated pointwise (and does not represent any type of function space norm). 
Definition 1.8. The degree of $p \in \mathbb{C}[z, w]$ will always refer to the bidegree. So,

$$
\operatorname{deg} p=(n, m)
$$

means $p$ has degree $n$ in $z$ and $m$ in $w$, while

$$
\operatorname{deg} p \leq(n, m)
$$

means $p$ has at most degree $n$ in $z$ and at most $m$ in $w$. The same notation applies to vector and matrix polynomials component-wise.

Frequent use will be made of the following notion of polynomial reflection.

Definition 1.9. If $p \in \mathbb{C}[z, w]$ is a polynomial of degree at most $(j, k)$ we define the reflection (at the $(j, k)$ degree $)$ to be

$$
\overleftarrow{p}(z, w):=z^{j} w^{k} \overline{p(1 / \bar{z}, 1 / \bar{w})}
$$

Remark 1.10. In the case of a stable polynomial (no zeros on the closed bidisk $\overline{\mathbb{D}^{2}}$ ), the theorem is deducible from the work of Geronimo and Woerdeman [2004]. It is the goal of the present paper to extend the sums of squares decomposition with uniqueness to all polynomials with no zeros on the open bidisk $\mathbb{D}^{2}$. Why are we concerned with such an extension?

First, it allows a direct, unified proof of the Cole-Wermer formula which does not make use of Andô's inequality, Agler's Pick interpolation theorem, or any of their close relatives (the original proof of Cole and Wermer relies heavily on these results). Our hope is that the uniqueness aspects could prove helpful in uniqueness issues of Pick interpolation on the bidisk.

Second, it allows us to improve a bounded analytic extension theorem (from [Knese 2009]) for the already alluded to curves called distinguished varieties. See Section 11.

Third, our method of proof may be of interest to some as we study orthogonal polynomials with respect to a positive Borel measure on $\mathbb{T}^{2}$ which may have infinite mass. Since such measures will not necessarily have finite moments, methods involving doubly Toeplitz matrices (as in [Geronimo and Woerdeman 2004]) are not directly available to us, and therefore our method of using reproducing kernels of subspaces of polynomials from [Knese 2008] is well adapted to the present situation. Our method of proof also allows us to improve a characterization of two-variable Fejér-Riesz factorizations from [Geronimo and Woerdeman 2004]. See Section 10.

Remark 1.11. The assumption that $q$ is almost stable (i.e., has finitely many zeros on $\mathbb{T}^{2}$ ) is there to put us into the most interesting case and not to avoid a difficulty. Every polynomial $q$ with no zeros on the bidisk can be factored into $q=q_{1} q_{2}$ where $q_{1}$ has at most finitely many zeros on the two-torus and every factor of $q_{2}$ has infinitely many zeros on the two-torus. If $q$ has a nontrivial factor of the type $q_{2}$, then it can be factored out of the entire sums of squares formula. These polynomials with no zeros on the bidisk and infinitely many zeros on the two-torus can be studied separately; see [Knese 2009]. These notions will appear several places later on so we give the following definitions of toral and atoral.

Definition 1.12. A polynomial $p \in \mathbb{C}[z, w]$ is toral if every factor of $p$ has infinitely many zeros on $\mathbb{T}^{2}$.

Definition 1.13. A polynomial $p \in \mathbb{C}[z, w]$ is atoral if $p$ has finitely many zeros on $\mathbb{T}^{2}$.

These terms were introduced in [Agler et al. 2006] in a more natural way that makes sense for higher dimensions, but these definitions will suffice for our purposes. 
Remark 1.14. The requirements on $\mathbf{E}$ and $\mathbf{F}$ in Theorem 1.3 that make the decomposition unique are essential in proving our bounded analytic extension theorem for distinguished varieties. The requirements are also curiously asymmetric. In fact, the entire formula (1-2) can be "reflected": replace $(z, w)$ with $(1 / \bar{z}, 1 / \bar{w})$ and multiply through by $-\left|z^{n} w^{m}\right|^{2}$. The result will be a new sums of squares formula with $\mathbf{E}$ and $\mathbf{F}$ replaced with

$$
\overleftarrow{\mathbf{E}}(z, w)=z^{n-1} w^{m} \overline{\mathbf{E}(1 / \bar{z}, 1 / \bar{w})} \quad \text { and } \quad \overleftarrow{\mathbf{F}}(z, w)=z^{n} w^{m-1} \overline{\mathbf{F}(1 / \bar{z}, 1 / \bar{w})}
$$

respectively. These new choices will have the stability requirements reversed in Theorem 1.3: E will be horizontally $\mathbb{E}$-stable and $\mathbf{F}$ will be vertically $\mathbb{D}$-stable. (Notice that in Example 2.1, below, the two choices for the sums of squares decompositions are not simply obtained from one another by performing this reflection.)

These thoughts beg the following question. Which almost stable polynomials have a unique sums of squares decomposition?

Theorem 1.15. Suppose $q \in \mathbb{C}[z, w]$ is almost stable with $\operatorname{deg} q \leq(n, m)$. The following are equivalent.

(1) There exist unique nonnegative functions $\Gamma_{1}, \Gamma_{2}$ which can be written as the sum of the squared moduli of two-variable polynomials such that

$$
|q(z, w)|^{2}-|\overleftarrow{q}(z, w)|^{2}=\left(1-|z|^{2}\right) \Gamma_{1}(z, w)+\left(1-|w|^{2}\right) \Gamma_{2}(z, w) .
$$

(2) There are no nonzero polynomials $f \in \mathbb{C}[z, w]$ with degree at most $(n-1, m-1)$ such that

$$
\frac{f}{q} \in L^{2}\left(\mathbb{T}^{2}\right)
$$

(3) There exist vector polynomials $\mathbf{E} \in \mathbb{C}^{n}[z, w], \operatorname{deg} \mathbf{E} \leq(n-1, m)$ and $\mathbf{F} \in \mathbb{C}^{m}[z, w], \operatorname{deg} \mathbf{F} \leq(n, m-1)$ satisfying

$$
|q(z, w)|^{2}-|\overleftarrow{q}(z, w)|^{2}=\left(1-|z|^{2}\right)|\mathbf{E}(z, w)|^{2}+\left(1-|w|^{2}\right)|\mathbf{F}(z, w)|^{2}
$$

that are symmetric in the sense that:

$$
\mathbf{E}(z, w)=z^{n-1} w^{m} \overline{\mathbf{E}(1 / \bar{z}, 1 / \bar{w})} \text { and } \mathbf{F}(z, w)=z^{n} w^{m-1} \overline{\mathbf{F}(1 / \bar{z}, 1 / \bar{w})} .
$$

Moreover, $\mathbf{E}$ is horizontally $\mathbb{D} \cup \mathbb{E}$-stable, and $\mathbf{F}$ is vertically $\mathbb{D} \cup \mathbb{E}$-stable.

The polynomial $q(z, w)=2-z-w$ from Example 1.1 has a unique sums of squares decomposition, since the decomposition we gave satisfies (3), after multiplying by a suitable unimodular constant. Item (2) says that the polynomials with a unique decomposition must in some sense have as many zeros as possible on the torus. Because of this, polynomials with no zeros on the closed bidisk never have unique decompositions unless they are one variable polynomials.

Corollary 1.16. If $q \in \mathbb{C}[z, w]$ is stable, then $q$ has a unique sums of squares decomposition if and only if $q$ is a function of only one variable (i.e., one of $q$ 's partial derivatives vanishes identically).

It would be interesting to have a parametrization of the polynomials in Theorem 1.15. Both Theorem 1.15 and Corollary 1.16 are proved in Section 9. 


\section{An example}

Example 2.1. Let $f(z, w)=2-z w-z^{2} w$. One decomposition of $f$ is

$$
\left|2-z w-z^{2} w\right|^{2}-\left|2 z^{3} w^{2}-z^{2} w-z w\right|^{2}=\left(1-|z|^{2}\right)|\mathbf{E}(z, w)|^{2}+\left(1-|w|^{2}\right)|\mathbf{F}(z, w)|^{2},
$$

where

$$
\begin{aligned}
& \mathbf{E}(z, w)=\sqrt{2}\left(\begin{array}{c}
1-z^{2} w \\
w-z w^{2} \\
z w-z^{2} w^{2}
\end{array}\right)=\sqrt{2}\left(\begin{array}{ccc}
1 & 0 & -w \\
w & -w^{2} & 0 \\
0 & w & -w^{2}
\end{array}\right)\left(\begin{array}{c}
1 \\
z \\
z^{2}
\end{array}\right), \\
& \mathbf{F}(z, w)=\sqrt{2}\left(\begin{array}{c}
z-z^{3} w \\
1-z w
\end{array}\right)=\sqrt{2}\left(\begin{array}{cc}
z & -z^{3} \\
1 & -z
\end{array}\right)\left(\begin{array}{c}
1 \\
w
\end{array}\right) .
\end{aligned}
$$

Alternatively, we could choose instead

$$
\begin{aligned}
& \mathbf{E}(z, w)=\left(\begin{array}{c}
\sqrt{2}\left(z-z^{2} w\right) \\
z-z^{2} \\
2-z w-z^{2} w
\end{array}\right)=\left(\begin{array}{ccc}
0 & \sqrt{2} & -\sqrt{2} w \\
0 & 1 & -1 \\
2 & -w & -w
\end{array}\right)\left(\begin{array}{c}
1 \\
z \\
z^{2}
\end{array}\right), \\
& \mathbf{F}(z, w)=\left(\begin{array}{c}
z+z^{2}-2 z^{3} w \\
z^{2}-z^{3}
\end{array}\right)=\left(\begin{array}{cc}
z+z^{2} & -2 z^{3} \\
z^{2}-z^{3} & 0
\end{array}\right)\left(\begin{array}{c}
1 \\
w
\end{array}\right) .
\end{aligned}
$$

These two choices are not equivalent up to unitary multiplication (reflecting is no remedy either) as can be checked. The second choices of $\mathbf{E}$ and $\mathbf{F}$ fit the requirements of Theorem 1.3, while the first choices do not.

\section{Sums of squares and uniqueness}

In this section we present several lemmas on sums of squares decompositions. Lemma 3.4 proves uniqueness in Theorem 1.3, namely, item (4). This section can easily be skipped and referred back to as necessary. It is included here because it does not require the more demanding notation of the rest of the paper.

The following theorem can be found in [D'Angelo 1993].

Theorem 3.1 (polarization for holomorphic functions). Let $\Omega$ be a domain in $\mathbb{C}^{N}$ and set

$$
\Omega^{*}=\left\{\bar{z}=\left(\bar{z}_{1}, \ldots, \bar{z}_{N}\right): z \in \Omega\right\} .
$$

If $f: \Omega \times \Omega^{*} \rightarrow \mathbb{C}$ is a holomorphic function with the property that

$$
f(z, \bar{z})=0 \quad \text { for all } z \in \Omega
$$

then

$$
f(z, w)=0 \quad \text { for all }(z, w) \in \Omega \times \Omega^{*} .
$$

The following lemma holds equally well for multivariable polynomials, and may be well known to some readers. See [Cole and Wermer 1999, Appendix] for a proof. 
Lemma 3.2. Suppose $\Gamma(z)$ is a sum of squares of polynomials and let $N$ be the rank of the matrix of coefficients of $\Gamma$. Then, there exists $\mathbf{A} \in \mathbb{C}^{N}[z]$ so that

$$
\Gamma(z)=|\mathbf{A}(z)|^{2}
$$

and $\mathbf{A}$ is minimal in the sense that

$$
|\mathbf{A}(z)|^{2} \equiv|\mathbf{B}(z)|^{2}, \quad \mathbf{B} \in \mathbb{C}^{M}[z]
$$

implies $\mathbf{B}(z)=V \mathbf{A}(z)$ for some isometric $M \times N$ matrix $V$.

Lemma 3.3. Let $\mathbf{E} \in \mathbb{C}^{n}[z, w], \operatorname{deg} \mathbf{E} \leq(n-1, m)$. Suppose $\mathbf{E}$ is horizontally $\mathbb{D}$-stable. Suppose further that $\mathbf{A} \in \mathbb{C}^{N}[z, w]$ satisfies

$$
|\mathbf{E}(z, w)|^{2}=|\mathbf{A}(z, w)|^{2} \quad \text { for }(z, w) \in \mathbb{C} \times \mathbb{T} .
$$

Then, $n \leq N, \mathbf{A}(z, w)$ has degree at most $n-1$ in $z$ and there exists an $N \times n$ matrix valued rational inner function $\Psi: \mathbb{D} \rightarrow \mathbb{C}^{N \times n}$, holomorphic on $\mathbb{D}$ such that

$$
\mathbf{A}(z, w)=\Psi(w) \mathbf{E}(z, w) .
$$

By $N \times n$ matrix valued inner function we mean that $\Psi$ is isometry valued on the circle (or more appropriately, unitary valued in the case $n=N$ ).

Proof. We have assumed

$$
|\mathbf{E}(z, w)|^{2}=|\mathbf{A}(z, w)|^{2},
$$

for all $z \in \mathbb{C}$ but $w \in \mathbb{T}$. By the polarization theorem for holomorphic functions

$$
\langle\mathbf{E}(z, w), \mathbf{E}(Z, w)\rangle=\langle\mathbf{A}(z, w), \mathbf{A}(Z, w)\rangle,
$$

for all $z, Z \in \mathbb{C}$ and $w \in \mathbb{T}$. The left hand side has degree at most $n-1$ in $z$ and this implies $\mathbf{A}(z, w)$ has degree at most $n-1$ in $z$ as follows. If some component with the largest degree, say $A_{1}(z, w)=$ $\sum_{j=0}^{M} a_{j}(w) z^{j}$, of $\mathbf{A}(z, w)$ has degree $M$ larger than $n-1$, then

$$
A_{1}(z, w) \overline{A_{1}(Z, w)}=\left|a_{M}(w)\right|^{2} z^{M} \bar{Z}^{M}+\text { lower order terms. }
$$

We necessarily have $a_{M}(w) \equiv 0$ on $\mathbb{T}$, which implies $a_{M}(w) \equiv 0$ for all $w \in \mathbb{C}$.

Therefore, we may write

$$
\mathbf{A}(z, w)=A(w) \Lambda_{n}(z),
$$

where $A(w)$ is an $N \times n$ matrix polynomial. Let us write

$$
\mathbf{E}(z, w)=E(w) \Lambda_{n}(z), \quad E(w) \in \mathbb{C}^{n \times n}[w] .
$$

Saying $\mathbf{E}$ is horizontally $\mathbb{D}$-stable means $E(w)$ is invertible for all $w \in \mathbb{D}$.

Rewriting (3-1) in matrix form we have

$$
\Lambda_{n}(Z)^{*} E(w)^{*} E(w) \Lambda_{n}(z)=\Lambda_{n}(Z)^{*} A(w)^{*} A(w) \Lambda_{n}(z),
$$

and since this holds for all $z, Z \in \mathbb{C}$

$$
E(w)^{*} E(w)=A(w)^{*} A(w)
$$


for all $w \in \mathbb{T}$ because $\Lambda_{n}(z)$ spans $\mathbb{C}^{n}$ as $z$ varies over any $n$ points. Now define

$$
\Psi(w)=A(w) E(w)^{-1}
$$

for $w \in \mathbb{D}$, a rational matrix polynomial with no poles on the disk (since $E(w)$ is invertible in the disk). Equation (3-2) says that $\Psi(w)$ is isometric for $w \in \mathbb{T}$. In particular, $n \leq N$, any singularities of $\Psi$ on the circle are removable ( $\Psi$ is rational and bounded on the circle), and by the maximum principle $\Psi$ is contraction valued in the disk. By definition, $\mathbf{A}(z, w)=\Psi(w) \mathbf{E}(z, w)$ for all $z, w \in \mathbb{C}$.

Lemma 3.4 (uniqueness). Let $\mathbf{E}, \tilde{\mathbf{E}} \in \mathbb{C}^{n}[z, w], \operatorname{deg} \mathbf{E}, \tilde{\mathbf{E}} \leq(n-1, m)$. Suppose both $\mathbf{E}$ and $\tilde{\mathbf{E}}$ are horizontally $\mathbb{D}$-stable.

Suppose further that there are vector polynomials $\mathbf{F}, \tilde{\mathbf{F}} \in \mathbb{C}^{m}[z, w]$ such that

$$
\left(1-|z|^{2}\right)|\mathbf{E}(z, w)|^{2}+\left(1-|w|^{2}\right)|\mathbf{F}(z, w)|^{2}=\left(1-|z|^{2}\right)|\tilde{\mathbf{E}}(z, w)|^{2}+\left(1-|w|^{2}\right)|\tilde{\mathbf{F}}(z, w)|^{2} .
$$

Then, there exists an $n \times n$ unitary $U_{1}$ and an $m \times m$ unitary $U_{2}$ such that

$$
\mathbf{E}(z, w)=U_{1} \tilde{\mathbf{E}}(z, w), \quad \mathbf{F}(z, w)=U_{2} \tilde{\mathbf{F}}(z, w) .
$$

Proof. Setting $|w|=1$ in (3-3) and canceling the factor $\left(1-|z|^{2}\right)$ we have

$$
|\mathbf{E}(z, w)|^{2}=|\tilde{\mathbf{E}}(z, w)|^{2} \quad \text { for }(z, w) \in \mathbb{C} \times \mathbb{T} .
$$

Both $\mathbf{E}$ and $\tilde{\mathbf{E}}$ satisfy the conditions of Lemma 3.3. Therefore, there exist $n \times n$ matrix valued rational inner functions $\Psi_{1}, \Psi_{2}: \mathbb{D} \rightarrow \mathbb{C}^{n \times n}$ such that

$$
\tilde{\mathbf{E}}(z, w)=\Psi_{1}(w) \mathbf{E}(z, w), \quad \mathbf{E}(z, w)=\Psi_{2}(w) \tilde{\mathbf{E}}(z, w) .
$$

This implies $\Psi_{1}(w) \Psi_{2}(w)=I$, and as $\Psi_{1}, \Psi_{2}$ are contractive valued, we must have $\Psi_{1}$ and $\Psi_{2}$ constant and equal to unitary matrices. Hence, there exists an $n \times n$ unitary matrix $U_{1}$ such that

$$
\mathbf{E}(z, w)=U_{1} \tilde{\mathbf{E}}(z, w)
$$

which implies

$$
|\mathbf{E}(z, w)|^{2}=|\tilde{\mathbf{E}}(z, w)|^{2} \quad \text { for all }(z, w) \in \mathbb{C}^{2} .
$$

In turn, by (3-3) we have

$$
|\mathbf{F}(z, w)|^{2}=|\tilde{\mathbf{F}}(z, w)|^{2} \quad \text { for all }(z, w) \in \mathbb{C}^{2} .
$$

By Lemma 3.2, there exists an $m \times m$ unitary matrix $U_{2}$ such that

$$
\mathbf{F}(z, w)=U_{2} \tilde{\mathbf{F}}(z, w) .
$$

We conclude this section with a lemma about the presence of zeros on the "undistinguished" portion of the boundary of $\mathbb{D}^{2}$, namely $(\mathbb{D} \times \mathbb{\mathbb { V }}) \cup(\mathbb{T} \times \mathbb{D})$.

Lemma 3.5. Suppose $q \in \mathbb{C}[z, w]$ has no zeros on $\mathbb{D}^{2}$. If $q\left(z_{0}, w_{0}\right)=0$ for some $\left(z_{0}, w_{0}\right) \in \mathbb{T} \times \mathbb{D}$, then $q\left(z_{0}, w\right)=0$ for all $w \in \mathbb{C}$; i.e., $\left(z-z_{0}\right)$ divides $q$. In particular, there can only be finitely many $z_{0} \in \mathbb{T}$ such that $q\left(z_{0}, \cdot\right)$ has a zero in $\mathbb{D}$. 
Proof. There is no harm in assuming $q$ is irreducible. Suppose $q\left(z_{0}, w\right)$ is not identically zero as a function of $w$. Then, we can apply the Weierstrass preparation theorem to $q$ and write

$$
q(z, w)=u(z, w)\left(z^{k}+a_{1}(w) z^{k-1}+\cdots+a_{k}(w)\right)
$$

on some bidisk $D_{1} \times D_{2}$ containing $\left(z_{0}, w_{0}\right)$ where $u$ is holomorphic and nonvanishing on $D_{1} \times D_{2}$ and each $a_{j}$ is holomorphic on $D_{2}$. We also assume $D_{2} \subset \mathbb{D}$. Furthermore, for $w \in D_{2} \backslash\left\{w_{0}\right\}$, each $a_{j}(w)$ is a symmetric function of the $k$ (necessarily) distinct roots (by irreducibility) $z_{1}(w), z_{2}(w), \ldots, z_{k}(w) \in D_{1}$ of $q(\cdot, w)$ for $w \in D_{2} \backslash\left\{w_{0}\right\}$. Note $a_{k}(w)=(-1)^{k} z_{1}(w) \cdots z_{k}(w)$ for $w \neq w_{0}$ and $a_{k}\left(w_{0}\right)=\left(-z_{0}\right)^{k}$. Since $q$ has no zeros in $\mathbb{D}^{2},\left|z_{j}(w)\right| \geq 1$ for all $j$ and $w \in D_{2}$, and hence $\left|a_{k}(w)\right| \geq 1$ for all $w \in D_{2}$. Since $\left|a_{k}\left(w_{0}\right)\right|=1$ the maximum principle implies $a_{k}$ is a unimodular constant, which in turn implies the roots $z_{1}(w), \ldots, z_{k}(w)$ are all unimodular valued. The roots must be constant and equal to $z_{0}$; that is, $q(z, w)$ can be divided by $z-z_{0}$.

\section{Preliminaries}

As in [Knese 2008], our approach will be to study two-variable orthogonal polynomials with respect to a positive Borel measure $\mu$ on the two-torus. The difference is that here we allow measures with infinite mass. In particular, we study "Bernstein-Szegő" measures on $\mathbb{T}^{2}$

$$
\frac{1}{|q(z, w)|^{2}} d \sigma
$$

where $d \sigma$ is normalized Lebesgue measure on the torus:

$$
d \sigma=d \sigma(z, w)=\frac{d z}{2 \pi i z} \frac{d w}{2 \pi i w},
$$

and $q \in \mathbb{C}[z, w]$ has finitely many zeros on $\mathbb{T}^{2}$ (and hence this measure can have infinite mass). On one hand, this causes a number of certain superficial (but still interesting) changes in the theory. For instance, we have to deal with the ideal $\mathbb{C}[z, w] \cap L^{2}(\mu)$ of polynomials in $L^{2}(\mu)$ as opposed to all of $\mathbb{C}[z, w]$ when studying orthogonal polynomials. (In particular, studying moment matrices will not be an option, because our measures may not have finite moments.) On the other hand, this change forces us to take greater care in certain situations. For instance, if $q \in \mathbb{C}[z, w]$ has no zeros on the bidisk and finitely many zeros on the two-torus, we cannot say (as we would in the case with no zeros on $\mathbb{T}^{2}$ ) that

$$
\int_{\mathbb{T}^{2}} \frac{1}{q(z, w)} d \sigma(z, w)=\frac{1}{q(0,0)}
$$

since $1 / q$ will not be integrable. Perhaps this integral could be understood in a principal value sense, however we confront this issue in our own way in Proposition 7.1.

Let us begin to provide some details. We shall make the following standing assumptions:

- $\mu$ is a positive Borel measure on $\mathbb{T}^{2}$;

- the ideal

$$
\Phi_{\mu}:=L^{2}(\mu) \cap \mathbb{C}[z, w]
$$

is nontrivial, where elements of $\mathbb{C}[z, w]$ here are thought of as measurable functions on $\mathbb{T}^{2}$; 
- the support of $\mu$ is not contained in the zero set of a nonzero polynomial, thus ensuring that $\|q\|_{L^{2}(\mu)} \neq 0$ if $q \neq 0$.

The inner product on $L^{2}(\mu)$ will be denoted by

$$
\langle f, g\rangle_{\mu}=\int_{\mathbb{T}^{2}} f \bar{g} d \mu .
$$

We shall make use of the machinery of reproducing kernel Hilbert spaces.

Notation 4.1. Given a finite-dimensional subspace $V \subset L^{2}(\mu) \cap \mathbb{C}[z, w]$, we shall use $K V$ to denote the reproducing kernel of $V$. Namely, for each $(Z, W) \in \mathbb{C}^{2}, K V_{(Z, W)}$ is the unique element of $V$ satisfying

$$
f(Z, W)=\left\langle f, K V_{(Z, W)}\right\rangle_{\mu} \quad \text { for all } f \in V
$$

and we define $K V: \mathbb{C}^{2} \times \mathbb{C}^{2} \rightarrow \mathbb{C}$ by

$$
K V((z, w),(Z, W)):=K V_{(Z, W)}(z, w) .
$$

It is not hard to show $K V$ is conjugate symmetric:

$$
K V((z, w),(Z, W))=\overline{K V((Z, W),(z, w))},
$$

and if $\left\{e_{1}, \ldots, e_{N}\right\}$ is an orthonormal basis of $V$, then

$$
K V((z, w),(Z, W))=\sum_{j=1}^{N} e_{j}(z, w) \overline{e_{j}(Z, W)} .
$$

Given $q \in \mathbb{C}[z, w]$ we use

$$
\hat{q}(j, k)
$$

to denote the coefficient of $z^{j} w^{k}$ in the Fourier series of $q$.

Remark 4.2. Throughout, we fix positive integers $n$ and $m$. The notations below depend on this.

We use the following notations as in [Knese 2008] which define subspaces of polynomials based on what frequencies may appear in their Fourier series (or in other language, we define subspaces based on the carrier of the polynomials). The symbols should be thought of a lying in the grid $\mathbb{Z}^{2}$ with the lower left corners representing the origin. A blackened section denotes excluded Fourier support. The box with the lower left corner missing $\square$ denotes the polynomials of degree at most $(n, m)$ which vanish at $(0,0)$, while the box with the upper right corner missing $\square$ denotes the polynomials of degree at most $(n, m)$ with no $(n, m)$ Fourier coefficient.

\section{Notation 4.3.}

$$
\begin{aligned}
& \square:=\{q \in \mathbb{C}[z, w]: \operatorname{deg}(q) \leq(n, m)\} \\
& \square:=\{q \in \mathbb{C}[z, w]: \operatorname{deg}(q) \leq(n-1, m)\} \\
& \square:=\{q \in \mathbb{C}[z, w]: \operatorname{deg}(q) \leq(n, m-1)\} \\
& \square:=\{q \in \mathbb{C}[z, w]: \operatorname{deg}(q) \leq(n-1, m-1)\} \\
& \square:=\{q \in \square: q(0,0)=0\} \\
& \square:=\{q \in \square: \hat{q}(n, m)=0\}
\end{aligned}
$$


For any of the above subspaces (and similar variations) we shall use a subscript $\mu$ to denote the intersection with $L^{2}(\mu)$. Namely,

$$
\begin{aligned}
\square_{\mu} & :=\square \cap L^{2}(\mu) \\
\square_{\mu} & :=\square \cap L^{2}(\mu) \\
\square_{\mu} & :=\square \cap L^{2}(\mu), \ldots .
\end{aligned}
$$

We use the following notations for shifts and certain orthogonal complements using the inner product on $L^{2}(\mu)$.

Notation 4.4.

$$
\begin{aligned}
& w \mathbf{\square}_{\mu}:=\left\{w p: p \in \mathbf{\square}_{\mu}\right\} \quad z \mathbf{\square}_{\mu}:=\left\{z p: p \in \mathbf{\square}_{\mu}\right\} \\
& \mathbf{\nabla}_{\mu}:=\square_{\mu} \ominus \mathbf{\square}_{\mu} \quad \boldsymbol{\Xi}_{\mu}:=\square_{\mu} \ominus\left(w \mathbf{\square}_{\mu}\right) \\
& \mathbf{\Psi}_{\mu}:=\square_{\mu} \ominus \square_{\mu} \quad \mathbb{\nabla}_{\mu}:=\square_{\mu} \ominus\left(z \mathbf{\square}_{\mu}\right) \\
& \square_{\mu}:=\square_{\mu} \ominus \square_{\mu} \quad \quad \square_{\mu}:=\square_{\mu} \ominus\left(w \square_{\mu}\right) \\
& \square_{\mu}:=\square_{\mu} \ominus \square_{\mu} \quad \text { 대 }:=\square_{\mu} \ominus \square_{\mu}
\end{aligned}
$$

For instance, $\mathbf{\Xi}_{\mu}$ denotes all $p \in \mathbb{C}[z, w] \cap L^{2}(\mu)$ of degree at most $(n-1, m)$ which are orthogonal to the polynomials in $\mathbb{C}[z, w] \cap L^{2}(\mu)$ of degree at most $(n-1, m-1)$.

A discussion of the notation. A more traditional notation for the subspaces above might work as follows:

$$
\begin{aligned}
\mathscr{P}_{n, m} & :=\{q \in \mathbb{C}[z, w]: \operatorname{deg}(q) \leq(n, m)\} \\
\mathscr{P}_{n, m-1} & :=\{q \in \mathbb{C}[z, w]: \operatorname{deg}(q) \leq(n, m-1)\} \\
\mathscr{P}_{n-1, m-1} & :=\{q \in \mathbb{C}[z, w]: \operatorname{deg}(q) \leq(n-1, m-1)\} \\
\mathscr{P}_{n, m}^{(n, m)} & :=\{q \in \mathbb{C}[z, w]: \operatorname{deg}(q) \leq(n, m), \hat{q}(n, m)=0\}
\end{aligned}
$$

In which case one could write out orthogonal complements in detail as in:

$$
\mathscr{P}_{n, m} \ominus \mathscr{P}_{n, m-1}
$$

To illustrate how cumbersome this becomes let us compare this more traditional notation with the box notation above. In the rest of this paper it will be important to decompose $\left(\mathscr{P}_{n, m}\right)_{\mu}$ (or $\square_{\mu}$ ) in a variety of ways. With more traditional notation we have:

$$
\begin{aligned}
& \mathscr{P}_{n, m}=\left(\mathscr{P}_{n, m} \ominus \mathscr{P}_{n, m}^{(n, m)}\right) \quad \oplus \mathscr{P}_{n, m}^{(n, m)} \\
& =\left(\mathscr{P}_{n, m} \ominus \mathscr{P}_{n, m}^{(n, m)}\right) \quad \oplus\left(\mathscr{P}_{n, m}^{(n, m)} \ominus \mathscr{P}_{n, m-1}\right) \oplus \mathscr{P}_{n, m-1} .
\end{aligned}
$$

All orthogonal sums and complements are taken with respect to $L^{2}(\mu)$. With our notation we have:

$$
\begin{aligned}
& \square_{\mu}=\underbrace{\left(\square_{\mu} \ominus \square_{\mu}\right)} \oplus \underbrace{\square_{\mu}} \\
& =\quad \Perp_{\mu} \oplus \quad \underbrace{\left(\square_{\mu} \ominus \square_{\mu}\right)} \oplus \square_{\mu} \\
& =\quad \square_{\mu} \oplus \quad \square \mu \quad \oplus \square_{\mu}
\end{aligned}
$$


It becomes necessary to take this even further:

$$
\begin{aligned}
& \square_{\mu}=\square_{\mu} \oplus \square_{\mu} \oplus \\
& =\square_{\mu} \oplus \quad \square_{\mu} \oplus \underbrace{\left(\square_{\mu} \ominus \boldsymbol{\square}_{\mu}\right)} \oplus \boldsymbol{\square}_{\mu} \\
& =\square_{\mu} \oplus \mathbb{\square}_{\mu} \oplus \quad \mathbb{\Xi}_{\mu} \oplus \mathbf{\square}_{\mu}
\end{aligned}
$$

Another way of decomposing $\square_{\mu}$ is as

$$
\square_{\mu}=\unlhd_{\mu} \oplus \unlhd_{\mu} \oplus \square_{\mu} \oplus \mathbf{\square}_{\mu}
$$

All of these decompositions translate into formulas for reproducing kernels since the reproducing kernel of a direct sum is the sum of the reproducing kernels [Knese 2008, Section 3]. Therefore,

$$
\begin{aligned}
& K \square_{\mu}=K \amalg_{\mu}+K \square_{\mu}+K \amalg_{\mu}+K \square_{\mu}, \\
& K \square_{\mu}=K \square_{\mu}+K \square_{\mu}+K \square_{\mu}+K \square_{\mu} .
\end{aligned}
$$

The two subspaces $\square_{\mu}, \Perp_{\mu}$ are either one-dimensional or trivial and are important enough to warrant special names:

$$
\begin{aligned}
& \operatorname{Max}_{\mu}:=\square_{\mu}=\square_{\mu} \ominus \square_{\mu}=\left\{p \in \mathbb{C}[z, w] \cap L^{2}(\mu): p \in \square_{\mu}, p \perp \square_{\mu}\right\}, \\
& \operatorname{Min}_{\mu}:=\square_{\mu}=\square_{\mu} \ominus \square_{\mu}=\left\{p \in \mathbb{C}[z, w] \cap L^{2}(\mu): p \in \square_{\mu}, p \perp \square_{\mu}\right\} .
\end{aligned}
$$

We choose these names because $p \in \operatorname{Max}_{\mu}$ maximizes the quantity

$$
\frac{|f(0,0)|}{\|f\|_{L_{\mu}^{2}}}
$$

among all $f \in \mathbb{C}[z, w] \cap L^{2}(\mu)$ of degree at most $(n, m)$. This follows from the fact that $p \in \operatorname{Max}_{\mu}$ if and only if $p$ is orthogonal to all $f \in \square_{\mu}$ vanishing at $(0,0)$. Elements of $\operatorname{Min}_{\mu}$ maximize the value of

$$
\frac{|\hat{f}(n, m)|}{\|f\|_{L_{\mu}^{2}}}
$$

among $f \in \square_{\mu}$.

We continue Example 1.1 to make all these definitions concrete.

Example 4.5. Let $q(z, w)=2-z-w$. Let

$$
d \mu=\frac{1}{|2-z-w|^{2}} d \sigma(z, w)=\frac{1}{(2 \pi i)^{2}|2-z-w|^{2}} \frac{d z}{z} \frac{d w}{w} .
$$

It turns out that $\Phi_{\mu}=L^{2}(\mu) \cap \mathbb{C}[z, w]$ equals the maximal ideal $(z-1, w-1) \subset \mathbb{C}[z, w]$. Indeed, a double application of Cauchy's formula shows

$$
1 \notin L^{2}(\mu) \text { and } z-1, w-1 \in L^{2}(\mu) .
$$


Also,

$$
2-z-w \perp w(z-1), z(w-1), \quad 2 z w-z-w \perp(z-1),(w-1) .
$$

If we set $n=1$ and $m=1$, then

$$
\begin{aligned}
& \square_{\mu}=\{0\}, \quad \text { since } 1 \notin L^{2}(\mu), \\
& \square_{\mu}=(w-1) \mathbb{C}, \\
& \square_{\mu}=(z-1) \mathbb{C}, \\
& \square_{\mu}=\operatorname{span}\{z-1, w-1, z+w-2 z w\}, \\
& \square_{\mu}=(2-z-w) \mathbb{C}, \\
& \square_{\mu}=(2 z w-z-w) \mathbb{C} .
\end{aligned}
$$

Since $\square_{\mu}$ is trivial,

$$
\mathbf{\Xi}_{\mu}=\square_{\mu} \ominus \mathbf{\square}_{\mu}=\square_{\mu} .
$$

In general, $\mathbf{\theta}_{\mu} \neq \square_{\mu}$, but the singularity of $\mu$ forces certain subspaces to degenerate.

\section{General properties of orthogonal polynomials on $\mathbb{t}^{2}$}

This section is about orthogonal polynomials on $\mathbb{T}^{2}$ with respect to a (not necessarily finite) positive Borel measure on $\mathbb{T}^{2}$. We use reproducing kernels to study entire subspaces of polynomials all at once, so the "orthogonal polynomials" are in some sense disguised.

The heart of the following two propositions should be familiar to those who know something about orthogonal polynomials on the unit circle. Namely, if $\rho$ is a probability measure on $\mathbb{T}$, and if $q \in$ $\mathbb{C}[z], \operatorname{deg} q \leq n$, then in $L^{2}(\rho)$

$$
q \perp z, z^{2}, \ldots, z^{n} \Longrightarrow q \text { is stable. }
$$

In two variables, consider the subspace of polynomials $\mathbb{\Xi}_{\mu}=\square_{\mu} \ominus \square_{\mu}$; that is, all

$$
p \in \mathbb{C}[z, w] \cap L^{2}(\mu), \operatorname{deg} p \leq(n, m-1)
$$

satisfying

$$
p \perp \operatorname{span}\left\{z^{j} w^{k}: 1 \leq j \leq n, 0 \leq k \leq m-1\right\} \cap L^{2}(\mu) .
$$

The conclusion of the first proposition below is that $p(z, w)$ has no factors of the form $\left(z-z_{0}\right)$ with $z_{0} \in \mathbb{D}$, and the second proposition says that a vector consisting of an orthonormal basis for $\mathbb{\Psi}_{\mu}$ is vertically $\mathbb{D}$-stable. Both of these notions are generalizations of one variable stability.

Another way to generalize orthogonal polynomials from one to two variables is to consider $p \in$ $\mathbb{C}[z, w] \cap L^{2}(\mu), \operatorname{deg} p \leq(n, m)$ satisfying

$$
p \perp \operatorname{span}\left\{z^{j} w^{k}: 0 \leq j \leq n, 0 \leq k \leq m,(j, k) \neq(0,0)\right\} \cap L^{2}(\mu),
$$

namely, $p \in \operatorname{Max}_{\mu}=\square_{\mu}$. This situation is much more subtle and is the topic of Section 6 .

Definition 5.1. We say an element $p$ of $\mathbb{C}[z, w]$ is a divisor of the ideal $\Phi_{\mu}$ if whenever $p q \in \mathscr{I}_{\mu}$, then $q \in \mathscr{I}_{\mu}$. 
Polynomials with no zeros on $\mathbb{T}^{2}$ are always divisors of $\mathscr{I}_{\mu}$.

\section{Proposition 5.2.}

(1) (a) If $p$ is a nonzero element of $\mathbb{\Psi}_{\mu}$ or $\Psi_{\mu}$, then $p$ is not divisible by a polynomial of the form $L(z, w)=z-z_{0}$ for $z_{0} \in \mathbb{D}$.

(b) If $p$ is a nonzero element of $\mathbb{\Xi}_{\mu}$ or $\Psi_{\mu}$ then $p$ is not divisible by any $L(z, w)=z-z_{0}$ when $z_{0} \in \mathbb{C} \backslash \overline{\mathbb{D}}$.

(c) In addition, if $z_{0} \in \mathbb{T}$, and $L(z, w)=z-z_{0}$ happens to be a divisor in $\Phi_{\mu}$, then nonzero elements

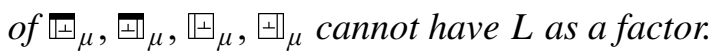

(2) (a) If $p$ is a nonzero element of $\Theta_{\mu}$ or $\Theta_{\mu}$, then $p$ cannot have a factor of the form $J(z, w)=w-w_{0}$ when $w_{0} \in \mathbb{D}$.

(b) If $p$ is a nonzero element of $\mathbf{\Xi}_{\mu}$ or $\mathbb{1}_{\mu}$, then $p$ cannot have a factor of the form $J(z, w)=w-w_{0}$ when $w_{0} \in \mathbb{C} \backslash \overline{\mathbb{D}}$.

(c) In addition, if $w_{0} \in \mathbb{T}$, and $J(z, w)=w-w_{0}$ happens to be a divisor in $\Phi_{\mu}$, then nonzero elements of $\Xi_{\mu}, \mathbf{\Xi}_{\mu}, \Theta_{\mu}, \mathbb{\Xi}_{\mu}$ cannot have $J$ as a factor.

Proof. We prove item (1a). Let $p \in \mathbb{\Psi}_{\mu}$ and suppose $p=g L$ for some $g \in \square$ where $L(z, w)=z-z_{0}$ with $\left|z_{0}\right|<1$. Since $L$ has no zeros on $\mathbb{T}^{2}, g=p / L \in L^{2}(\mu)$. Then, $z_{0} g(z, w)=z g(z, w)-p(z, w)$ and

$$
\left|z_{0}\right|^{2}\|g\|_{L^{2}(\mu)}^{2}=\|-p+z g\|_{L^{2}(\mu)}^{2}=\|p\|_{L^{2}(\mu)}^{2}+\|z g\|_{L^{2}(\mu)}^{2}=\|p\|_{L^{2}(\mu)}^{2}+\|g\|_{L^{2}(\mu)}^{2} .
$$

since $p \perp_{\mu} z g$. Rearranging we arrive at

$$
\|p\|_{L^{2}(\mu)}^{2}=\left(\left|z_{0}\right|^{2}-1\right)\|g\|_{L^{2}(\mu)}^{2}<0,
$$

a contradiction. The proofs of the other statements are variations on the above idea.

Curiously, slightly more complicated factors can be ruled out by a similar argument. For instance, if $|a|<1$, then $P(z, w)=z^{2}-a w^{3}$ cannot be a factor of any polynomial in $\square_{\mu}$. If $|a|=1$ and $P$ is a divisor of $\mathscr{I}_{\mu}$ then the same conclusion holds.

The next proposition shows that horizontal $\mathbb{D}$-stability occurs naturally (recall Definition 1.6).

Proposition 5.3. Let $\left\{e_{1}, \ldots, e_{N}\right\} \subset \mathbb{C}[z, w]$ be an orthonormal basis for $\boldsymbol{\theta}_{\mu}$ which we write vectorially as $\mathbf{E}(z, w)=\left(e_{1}(z, w), \ldots, e_{N}(z, w)\right)^{t}$. Then, $N \leq n$ and $\mathbf{E}$ is horizontally $\mathbb{D} \cup X$-stable, where $X \subset \mathbb{\mathbb { V }}$ is the set of $w_{0} \in \mathbb{T}$ such that $L(z, w)=w-w_{0}$ is a divisor of $\Phi_{\mu}$.

The same results hold for $\mathbb{\Psi}_{\mu}$ with the roles of $z$ and $w$ switched.

Proof. First, we claim $\operatorname{dim} \Theta_{\mu}:=N \leq n$. Given $n+1$ polynomials in $\Theta_{\mu}$, some linear combination of them will be a multiple of $w$ (since the degree in $z$ is at most $n-1$ ); such a combination would be orthogonal to itself (by definition of $\Theta_{\mu}$ ) and therefore zero; and hence any $n+1$ polynomials in $\boldsymbol{\Theta}_{\mu}$ are dependent. So, $\operatorname{dim} \boldsymbol{\theta}_{\mu} \leq n$.

Write

$$
\mathbf{E}(z, w)=E(w) \Lambda_{n}(z),
$$

where $E(w)$ is an $(N \times n)$-matrix valued polynomial in $w$ of degree at most $m$. We must prove $\mathbf{E}$ is horizontally $\mathbb{D} \cup X$-stable which means $E(w)$ has rank $N$ for all $w \in \mathbb{D} \cup X$. 
So, suppose $E\left(w_{0}\right)$ has rank less than $N$ at some point $w_{0} \in \mathbb{C}$. Since $E\left(w_{0}\right)$ is $N \times n$ and $N \leq n$ there must be a nonzero vector $\mathbf{v} \in \mathbb{C}^{N}$ such that $\mathbf{v}^{t} E\left(w_{0}\right)=\mathbf{0}^{t}$; that is, the following (necessarily nonzero) polynomial

$$
q(z, w)=\mathbf{v}^{t} E(w) \Lambda_{n}(z)=\mathbf{v}^{t} \mathbf{E}(z, w)
$$

is in $\Theta_{\mu}$ and vanishes on the set $\left\{w=w_{0}\right\}$. By the previous proposition this can only happen if $w_{0} \notin \mathbb{D}$ and when $w_{0} \in \mathbb{T}, w-w_{0}$ cannot be a divisor of $\mathscr{I}_{\mu}$. So, $E\left(w_{0}\right)$ has full rank $N$ everywhere in $\mathbb{D}$ and at all points $w_{0} \in \mathbb{\mathbb { V }}$ for which $w-w_{0}$ is a divisor of $\mathscr{I}_{\mu}$; that is, $\mathbf{E}$ is horizontally $\mathbb{D} \cup X$-stable.

Continuing our previous aside, we can also say that $\mathbf{E} \in \mathbb{C}^{N}[z, w]$ as above when restricted to the variety $\left\{z^{2}-a w^{3}=0\right\}$ (here $|a|<1$ ) does not sit inside any proper subspace of $\mathbb{C}^{N}$.

Remark 5.4. The main ideas of the previous two propositions appeared in the appendix of [Knese 2009] in a less detailed form.

The following is an analogue of the one variable Christoffel-Darboux formula.

Proposition 5.5 (Christoffel-Darboux type formulas). Suppressing $((z, w),(z, w))$ in front of each kernel we have

$$
K \varpi_{\mu}-K \mathbf{\Xi}_{\mu}=\left(1-|z|^{2}\right) K \mathbf{\square}_{\mu} \quad \text { and } \quad K \boldsymbol{\Xi}_{\mu}-K \mathbf{\Xi}_{\mu}=\left(1-|w|^{2}\right) K \mathbf{\square}_{\mu} \text {. }
$$

Proof. Let us decompose $\square_{\mu}$, the subspace of polynomials $p \in \mathbb{C}[z, w] \cap L^{2}(\mu)$, deg $p \leq(n, m-1)$, in two ways:

$$
\begin{aligned}
& \square_{\mu}=\left(\square_{\mu} \ominus \square_{\mu}\right) \oplus \square_{\mu}=\square_{\mu} \oplus z \square_{\mu}, \\
& \square_{\mu}=\left(\square_{\mu} \ominus \square_{\mu}\right) \oplus \square_{\mu}=\square_{\mu} \oplus \mathbf{\square}_{\mu} .
\end{aligned}
$$

The reproducing kernel of a direct sum is the sum of the reproducing kernels [Knese 2008, Section 3], and so

$$
\begin{aligned}
& K \mathbb{\boxplus}_{\mu}+\underbrace{K\left(z \mathbf{\square}_{\mu}\right)}=K \mathbf{\Psi}_{\mu}+K \mathbf{\square}_{\mu}, \\
& K \mathbb{\boxplus}_{\mu}+z \bar{Z} K \mathbf{\square}_{\mu}=K \mathbf{\Psi}_{\mu}+K \mathbf{\square}_{\mu},
\end{aligned}
$$

since shifting a subspace by $z$ "shifts" the reproducing kernel by the factor $z \bar{Z}$. Here we have suppressed the argument $((z, w),(Z, W))$ in front of every reproducing kernel. After rearranging we get the first equation of the proposition:

$$
K \square_{\mu}-K \mathbf{\Psi}_{\mu}=K \mathbf{\square}_{\mu}-z \bar{Z} K \square_{\mu}=(1-z \bar{Z}) K \square_{\mu} .
$$

The proof of the second equation is similar.

Definition 5.6. A polynomial $p \in \mathbb{C}[z, w]$ is $\mathbb{T}^{2}$-symmetric if it equals a unimodular constant $\mu$ times its reflection:

$$
p(z, w)=\mu \overleftarrow{p}(z, w)=\mu z^{j} w^{k} \overline{p(1 / \bar{z}, 1 / \bar{w})}
$$

here $p$ has degree exactly $(j, k)$.

Proposition 5.7. Let $P$ be the greatest common divisor of $\square_{\mu}$. Then, every factor of $P$ is $\mathbb{T}^{2}$-symmetric and the zero set of every factor of $P$ intersects $\mathbb{T}^{2}$. 
Proof. The greatest common divisor $P$ is necessarily $\mathbb{T}^{2}$-symmetric (basically since the set $\square_{\mu}$ is). Let $q$ be an irreducible factor of $P$ and let $j$ be the highest power such that $q^{j}$ divides $P$. Suppose $q$ is not a multiple of $\overleftarrow{q}$. Then $q^{j} \overleftarrow{q}^{j}$ divides $P$. Let $p$ be an element of $\square_{\mu}$ divisible by the maximal number of factors of $q$; that is, $q^{k}$ divides $p$ and no nonzero element of $\square_{\mu}$ is divisible by $q^{k+1}$. Since $\overleftarrow{q}^{j}$ divides $p$ we may write $p=q^{k} \overleftarrow{q}^{j} g$ for some $g \in \mathbb{C}[z, w]$. Since $|q|=|\overleftarrow{q}|$ on $\mathbb{T}^{2}$, it follows that $p$ being in $L^{2}(\mu)$ implies $q^{k+j} g \in L^{2}(\mu)$. In particular, $q^{k+j} g \in \square_{\mu}$ contradicting the maximality property of $p$ and $k$. Hence, $q$ must be $\mathbb{T}^{2}$-symmetric.

The zero set of every factor $q$ of $P$ must intersect $\mathbb{T}^{2}$ since otherwise $q g \in L^{2}(\mu)$ implies $g \in L^{2}(\mu)$ for any $g \in \mathbb{C}[z, w]$.

Question 5.8. Is $P$ toral? That is, does the zero set of every factor of $P$ intersect $\mathbb{T}^{2}$ on an infinite set?

This question is made more difficult by the fact that there exist irreducible, atoral, $\mathbb{T}^{2}$-symmetric polynomials:

$$
p(z, w)=(3 z+1) w^{2}-(z+3)(3 z+1) w+z(z+3)
$$

is such a polynomial taken from [Agler et al. 2008].

\section{OC measures}

The following theorem should be thought of as an attempt to prove a two-variable Christoffel-Darboux formula for general positive Borel measures which fails. The expression $\epsilon$ below measures how much it fails.

Theorem 6.1. Let $\mu$ be a positive Borel measure on $\mathbb{T}^{2}$ for which $\mathbb{C}[z, w] \cap L^{2}(\mu) \neq\{0\}$ and for which $\operatorname{Max}_{\mu}=\rrbracket_{\mu}$ is one-dimensional. Let

$$
\epsilon:=\left(K \amalg_{\mu}-K \text { 田 }_{\mu}\right)-\left(K \square_{\mu}-K \Xi_{\mu}\right) .
$$

If $q$ is any unit norm polynomial in $\operatorname{Max}_{\mu}$, then writing

$$
q \bar{q}=q(z, w) \overline{q(Z, W)}
$$

and omitting the expressions $((z, w),(Z, W))$, we get:

$$
\begin{aligned}
& q \bar{q}-\stackrel{\leftarrow}{q} \bar{q}=(1-z \bar{Z})(1-w \bar{W}) K \mathbf{\square}_{\mu} \\
& +(1-z \bar{Z}) K \text { 龱 }_{\mu}+(1-w \bar{W}) K \text { 凷 }_{\mu}+\epsilon \\
& =(1-z \bar{Z}) K \text { 国 }_{\mu}+(1-w \bar{W}) K \mathbb{\boxplus}_{\mu}+\epsilon \\
& =(1-z \bar{Z}) K \text { 田 }_{\mu}+(1-w \bar{W}) K \text { 四 }_{\mu}+\epsilon \text {. }
\end{aligned}
$$

The proof of this theorem is identical to the proof of Theorem 4.5 in [Knese 2008], which is for probability measures. We already have many of the details in place so it seems worthwhile to include the proof. 
Proof. By Equation (4-5),

$$
\begin{aligned}
& K \square_{\mu}=K \amalg_{\mu}+K \text { ت }_{\mu}+K \text { 田 }_{\mu}+K \square_{\mu}
\end{aligned}
$$

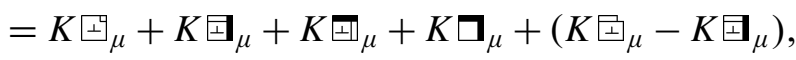

and, by Equation (4-6),

$$
\begin{aligned}
& K \square_{\mu}=K \square_{\mu}+K \square_{\mu}+K \unlhd_{\mu}+K \square_{\mu} \\
& =K \varpi_{\mu}+K \Xi_{\mu}+K \varpi_{\mu}+K \mathbf{\square}_{\mu}+\left(K \varpi_{\mu}-K \Xi_{\mu}\right) \\
& =K \square_{\mu}+z \bar{Z} K \Theta_{\mu}+w \bar{W} K \square_{\mu}+z \bar{Z} w \bar{W} K \square_{\mu}+\left(K \sqcup_{\mu}-K \Xi_{\mu}\right) \text {. }
\end{aligned}
$$

Using the formulas in Proposition 5.5 to eliminate $K \mathbb{\Psi}_{\mu}$ and $K \Theta_{\mu}$, we get:

$$
\begin{aligned}
& K \square_{\mu}=K \square_{\mu}+z \bar{Z}\left(K \mathbf{\Xi}_{\mu}+(1-w \bar{W}) K \mathbf{\square}_{\mu}\right)+w \bar{W}\left(K \mathbf{⿴ 囗 十}_{\mu}+(1-z \bar{Z}) K \mathbf{\square}_{\mu}\right) \\
& +z \bar{Z} w \bar{W} K \square_{\mu}+\left(K \bigsqcup_{\mu}-K \Xi_{\mu}\right) \\
& =K \square_{\mu}+z \bar{Z} K \text { 田 }_{\mu}+w \bar{W} K \text { 田 }_{\mu} \\
& +(z \bar{Z}+w \bar{W}-z \bar{Z} w \bar{W}) K \square_{\mu}+\left(K \unlhd_{\mu}-K \Xi_{\mu}\right) .
\end{aligned}
$$

Combined with Equation (6-1) above we have

$$
K \amalg_{\mu}-K \amalg_{\mu}=(1-z \bar{Z}) K \text { 田 }_{\mu}+(1-w \bar{W}) K \amalg_{\mu}+(1-z \bar{Z})(1-w \bar{W}) K \mathbf{\square}_{\mu}+\epsilon .
$$

Note that since $\operatorname{Max}_{\mu}=\square_{\mu}$ is one-dimensional, $q \bar{q}$ is its reproducing kernel. Likewise, $\operatorname{Min}_{\mu}=\amalg_{\mu}$ is the reflection of $\operatorname{Max}_{\mu}$ and therefore has reproducing kernel $\stackrel{q}{q}$. This proves the first formula of the theorem.

The remaining formulas follow from Proposition 5.5 by eliminating either $K \mathbb{\Psi}_{\mu}$ or $K \mathbf{\Xi}_{\mu}$. See [Knese 2008] for more details.

The $\epsilon$ in Theorem 6.1 is identically zero for measures of the following type, as we explain below.

Definition 6.2. We will call the measure $\mu$ an $O C$ measure if it satisfies this orthogonality condition:

$$
\square_{\mu}=\text { 田 }_{\mu} \text {. }
$$

These measures are so fundamental to the rest of the paper that they warrant extra discussion. Note that being an OC measure is only a constraint on how $\mu$ behaves with respect to polynomials of degree at most $(n, m)$. When $\mu$ is a finite measure, being an OC measure is a condition on the moments of $\mu$, as is explained in [Knese 2008, Appendix].

Discussion of OC measures. Recall $\Phi_{\mu}=\mathbb{C}[z, w] \cap L^{2}(\mu)$. Here are four ways to interpret OC measures:

- Every $p \in \mathscr{I}_{\mu}$ of degree at most $(n, m)$ with $\hat{p}(n, m)=0$ which is orthogonal to polynomials in $\mathscr{I}_{\mu}$ of degree at most $(n, m-1)$ automatically satisfies

$$
\hat{p}(n, k)=0 \text { for } k=0,1, \ldots, m-1 .
$$

In symbols:

$$
\left(p \in \square_{\mu} \text { and } p \perp \square_{\mu}\right) \Longrightarrow p \in \square_{\mu}
$$


- Every $p \in \Phi_{\mu}$ of degree at most $(n-1, m)$ which is orthogonal to all polynomials in $\Phi_{\mu}$ of degree at most $(n-1, m-1)$ is automatically orthogonal to all polynomials in $\Im_{\mu}$ of degree at most $(n, m-1)$. In symbols:

$$
\left(p \in \square_{\mu} \text { and } p \perp \mathbf{\square}_{\mu}\right) \Longrightarrow p \perp \square_{\mu} .
$$

- An OC measure satisfies a certain inclusion-exclusion principle:

$$
0=K \square_{\mu}-K \square_{\mu}-K \square_{\mu}+K \square_{\mu} .
$$

To see this, consider the decompositions

$$
\begin{aligned}
& K \square_{\mu}=K \text { घ }_{\mu}+K \varpi_{\mu}+K \square_{\mu}, \\
& K \square_{\mu}=K \text { 田 }_{\mu}+K \square_{\mu}, \\
& K \square_{\mu}=K \varpi_{\mu}+K \square_{\mu} .
\end{aligned}
$$

When $\mu$ is an OC measure, $\Xi_{\mu}=\Xi_{\mu}$. This yields Equation (6-2).

The symmetry in Equation (6-2) also proves that

$$
\square_{\mu}=\Xi_{\mu} \quad \text { if and only if } \varpi_{\mu}=\Psi_{\mu} .
$$

- An OC measure behaves like a Bernstein-Szegố measure

$$
\frac{1}{|q(z, w)|^{2}} d \sigma(z, w)
$$

here $q \in \mathbb{C}[z, w]$ has no zeros on $\mathbb{D}^{2}$. Section 7 is devoted to this fact and its converse: BernsteinSzegő measures are OC measures! See Corollary 7.6 and Theorem 7.4.

Additionally, if $\Xi_{\mu}=\mathbf{\theta}_{\mu}$ holds, then we have

$$
\square^{\square}=\Xi_{\mu}
$$

by reflecting these subspaces (polynomial reflection is an antiunitary and so preserves orthogonality relations).

Therefore, if $\mu$ is an OC measure then the $\epsilon$ in Theorem 6.1, given by $\left(K \Xi_{\mu}-K \mathbb{\Xi}_{\mu}\right)-\left(K \square_{\mu}-K \mathbb{\Xi}_{\mu}\right)$, disappears.

Hence, if $\mu$ is an OC measure, we have

$q(z, w) \overline{q(Z, W)}-\overleftarrow{q}(z, w) \bar{q} \overline{q(Z, W)}=(1-z \bar{Z}) K$ 龱 $_{\mu}((z, w),(Z, W))+(1-w \bar{W}) K \mathbb{\varpi}_{\mu}((z, w),(Z, W))$,

where $q$ is any unit norm polynomial in $\operatorname{Max}_{\mu}=\square_{\mu}$.

Evaluating on the diagonal $(z, w)=(Z, W)$ we have

$$
\begin{aligned}
|q(z, w)|^{2} & \geq|q(z, w)|^{2}-|\overleftarrow{q}(z, w)|^{2} \\
& =\left(1-|z|^{2}\right) K \mathbb{\Xi}_{\mu}((z, w),(z, w))+\left(1-|w|^{2}\right) K \varpi_{\mu}((z, w),(z, w)) \geq 0,
\end{aligned}
$$

for all $(z, w) \in \overline{\mathbb{D}^{2}}$. If we scrutinize this inequality, we can prove something quite strong. 
Proposition 6.3. Let $\mu$ be an OC measure and let $q$ be any unit norm polynomial in $\operatorname{Max}_{\mu}$. If $q\left(z_{0}, w_{0}\right)$ vanishes for some $\left(z_{0}, w_{0}\right) \in \overline{\mathbb{D}^{2}}$, every element of $\square_{\mu}$ vanishes at $\left(z_{0}, w_{0}\right)$.

Proof. Recall two formulas from above. By Proposition 5.5

$$
K \mathbb{\Psi}_{\mu}-K \mathbf{\Psi}_{\mu}=\left(1-|z|^{2}\right) K \mathbf{\square}_{\mu}
$$

and by (4-5)

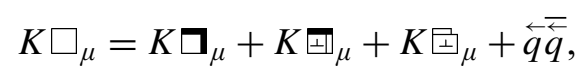

where every reproducing kernel is evaluated on the diagonal $(z, w)=(Z, W)$.

First, suppose $\left(z_{0}, w_{0}\right) \in \mathbb{D}^{2}$. We write $v=\left(z_{0}, w_{0}\right)$ for short. From $(6-3)$, it is immediate that $q(v)=0$ implies

$$
\overleftarrow{q}(v)=K \mathbf{\Xi}_{\mu}(v, v)=K \mathbb{\Psi}_{\mu}(v, v)=0
$$

Then, $K \square_{\mu}(v, v)=0$ by formulas (6-4) and (6-5). Indeed, $K \mathbb{\Psi}_{\mu}(v, v)=0$ implies $K \mathbf{\Psi}_{\mu}(v, v)=$ $K \boldsymbol{\square}_{\mu}(v, v)=0$ by (6-4) (using the fact that reproducing kernels are nonnegative on the diagonal). Then, (6-5) implies $K \square_{\mu}(v, v)=0$ since $K \square_{\mu}=K \mathbf{\Xi}_{\mu}$ by assumption. If $K \square_{\mu}(v, v)=0$ then every element of $\square \mu$ must vanish at $v$.

To prove the claim for $v=\left(z_{0}, w_{0}\right) \in \overline{\mathbb{D}^{2}} \backslash \mathbb{D}^{2}$, notice that the left hand side of (6-3) vanishes to order at least two at $v$, and the terms $\left(1-|z|^{2}\right)$ and $\left(1-|w|^{2}\right)$ can vanish to order at most one. This again implies (6-6) and by a similar argument $K \square_{\mu}(v, v)=0$.

Therefore, every element of $\square_{\mu}$ vanishes at a zero of $q$ in $\overline{\mathbb{D}^{2}}$.

Remark 6.4. If $\mu$ is a finite measure, then $1 \in \square_{\mu}$ and this implies $q$ has no zeros on the closed bidisk. Hence, this proves stability in the case of probability measures, as in [Geronimo and Woerdeman 2004; Knese 2008].

Corollary 6.5. Suppose $\mu$ is an OC measure and let $q$ be any unit norm polynomial in $\operatorname{Max}_{\mu}$. Then, $q$ can be factored into $q=q_{1} q_{2}$ where

- $q_{1}$ divides every element of $\square_{\mu}$;

- every irreducible factor of $q_{1}$ is $\mathbb{T}^{2}$-symmetric, has infinitely many zeros in $\overline{\mathbb{D}^{2}}$, and vanishes somewhere on $\mathbb{T}^{2}$; and

- $q_{2}$ has no zeros in $\overline{\mathbb{D}^{2}} \backslash \mathbb{T}^{2}$ and finitely many zeros in $\mathbb{T}^{2}$.

Proof. It is clear $q$ may be factored into the form $q=q_{1} q_{2}$ where every irreducible factor of $q_{1}$ has infinitely many zeros in $\overline{\mathbb{D}^{2}}$ and $q_{2}$ has finitely many zeros in $\overline{\mathbb{D}^{2}}$ (we of course allow for the case where $q_{1}$ or $q_{2}$ is a constant).

Suppose $f$ is an irreducible factor of $q$ possessing infinitely many zeros in $\overline{\mathbb{D}^{2}}$; that is, a factor of $q_{1}$. By Proposition 6.3, every element of $\square_{\mu}$ has infinitely many zeros in common with $f$ and hence $f$ divides every element of $\square_{\mu}$. So, $f$ can be divided out of both sides of the inequality (6-3) and using the resulting inequality one can then show that if $f$ occurs in the factorization of $q$ with multiplicity, it then divides every element of $\square_{\mu}$ with the same multiplicity. Hence, $q_{1}$ divides every element of $\square_{\mu}$. By Proposition 5.7, any such $f$ necessarily is $\mathbb{T}^{2}$-symmetric and vanishes somewhere on $\mathbb{T}^{2}$. This proves the first two items in the statement of the corollary. 
Finally, if $q_{2}$ has finitely many zeros in $\overline{\mathbb{D}^{2}}, q_{2}$ can have no zeros in the bidisk. By Lemma $3.5, q_{2}$ can have no zeros on the sides: $\mathbb{D} \times \mathbb{T}$ and $\mathbb{T} \times \mathbb{D}$. This proves the third item.

Since the factor $q_{1}$ in the above corollary divides every element of $\square_{\mu}$, the study of $\mu$ and $\square_{\mu}$ can be separated into the study of $q_{1}$ and the study of $\left|q_{1}\right|^{2} d \mu$ and the set $\square_{\mu} / q_{1}$ (which is nothing more than all $p \in L^{2}\left(\left|q_{1}\right|^{2} d \mu\right)$ of degree less than or equal to $\left(n-n_{1}, m-m_{1}\right)$, where $\left(n_{1}, m_{1}\right)$ is the degree of $\left.q_{1}\right)$. Indeed, the map sending

$$
f \in \square_{\mu} \mapsto f / q_{1} \in \square_{\mu} / q_{1}
$$

is an isometry (using the inner product of $L^{2}(\mu)$ on the left and the inner product of $L^{2}\left(\left|q_{1}\right|^{2} d \mu\right)$ on the right). Although this is a somewhat trivial observation, we now feel justified in making the assumption that $\operatorname{Min}_{\mu}$ and $\operatorname{Max}_{\mu}$ have no common factor, a statement equivalent to saying $q$ and $\overleftarrow{q}$ have no common factor. A statement which is in turn equivalent to saying $q_{1}$ is a constant. The following proposition is immediate, since its hypotheses imply $q=q_{2}$ in Corollary 6.5 .

Proposition 6.6. If $\mu$ is an OC measure and if $\operatorname{Max}_{\mu}$ and $\operatorname{Min}_{\mu}$ are one-dimensional and have no factor in common, then any $q \in \operatorname{Max}_{\mu}$ is almost stable.

Lemma 6.7. Suppose $\operatorname{Min}_{\mu}$ is one-dimensional and has no factor in common with $\mathrm{Max}_{\mu}$, and suppose $\mu$ is an OC measure. Then,

$$
\operatorname{dim} \Theta_{\mu}=n \quad \text { and } \quad \operatorname{dim} \Psi_{\mu}=m \text {. }
$$

Proof. Let $h$ be a unit norm polynomial in $\operatorname{Min}_{\mu}$. The polynomial $h$ necessarily has degree exactly $(n, m)$, otherwise it would be orthogonal to itself. Set $q=\overleftarrow{h}$, where the reflection is performed at the $(n, m)$ level. By Theorem 6.1 with $\epsilon=0$,

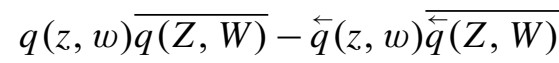

$$
=(1-z \bar{Z}) K \text { 四 }_{\mu}((z, w),(Z, W))+(1-w \bar{W}) K \text { 龱 }_{\mu}((z, w),(Z, W)) .
$$

Let $d_{1}=\operatorname{dim} \Theta_{\mu}$ and $d_{2}=\operatorname{dim} \Psi_{\mu}$; let $e_{1}, \ldots, e_{d_{1}}$ be an orthonormal basis for $\Theta_{\mu}$ and $f_{1}, \ldots, f_{d_{2}}$ an orthonormal basis for $\mathbb{I}_{\mu}$. We write these vectorially as

$$
\mathbf{E}(z, w)=\left(\begin{array}{c}
e_{1}(z, w) \\
\vdots \\
e_{d_{1}}(z, w)
\end{array}\right) \quad \text { and } \quad \mathbf{F}(z, w)=\left(\begin{array}{c}
f_{1}(z, w) \\
\vdots \\
f_{d_{2}}(z, w)
\end{array}\right)
$$

and then the formula above becomes

$$
q(z, w) \overline{q(Z, W)}-\overleftarrow{q}(z, w) \bar{q}(Z, W)=(1-z \bar{Z})\langle\mathbf{E}(z, w), \mathbf{E}(Z, W)\rangle+(1-w \bar{W})\langle\mathbf{F}(z, w), \mathbf{F}(Z, W)\rangle
$$

Upon rearranging we have

$$
\begin{aligned}
& q(z, w) \overline{q(Z, W)}+z \bar{Z}\langle\mathbf{E}(z, w), \mathbf{E}(Z, W)\rangle+w \bar{W}\langle\mathbf{F}(z, w), \mathbf{F}(Z, W)\rangle \\
& =\overleftarrow{q}(z, w) \overleftarrow{q}(Z, W)+\langle\mathbf{E}(z, w), \mathbf{E}(Z, W)\rangle+\langle\mathbf{F}(z, w), \mathbf{F}(Z, W)\rangle
\end{aligned}
$$


The map defined by

$$
\left(\begin{array}{c}
q(z, w) \\
z \mathbf{E}(z, w) \\
w \mathbf{F}(z, w)
\end{array}\right) \mapsto\left(\begin{array}{c}
\overleftarrow{q}(z, w) \\
\mathbf{E}(z, w) \\
\mathbf{F}(z, w)
\end{array}\right)
$$

for each $(z, w) \in \mathbb{C}^{2}$ defines a unitary on the span of the elements in $\mathbb{C}^{1+d_{1}+d_{2}}$ of the form on the left to the span of the elements in $\mathbb{C}^{1+d_{1}+d_{2}}$ of the form on the right, which can be extended to a unitary matrix $U$ of dimensions $\left(1+d_{1}+d_{2}\right) \times\left(1+d_{1}+d_{2}\right)$. We write $U$ in block form as

$$
U=\begin{aligned}
& \mathbb{C} \\
& \mathbb{C} \\
& \mathbb{C}^{d_{1}+d_{2}}\left(\begin{array}{ll}
A & B \\
C & D
\end{array}\right)
\end{aligned}
$$

We also define a $\mathbb{C}^{d_{1}+d_{2}}$-valued polynomial $\mathbf{G}$ by

$$
\mathbf{G}(z, w):=\left(\begin{array}{l}
\mathbf{E}(z, w) \\
\mathbf{F}(z, w)
\end{array}\right)
$$

and define the $\left(d_{1}+d_{2}\right) \times\left(d_{1}+d_{2}\right)$ diagonal matrix

$$
\Delta(z, w):=\left(\begin{array}{cc}
z I_{d_{1}} & 0 \\
0 & w I_{d_{2}}
\end{array}\right)
$$

Then,

$$
\begin{aligned}
& A q(z, w)+B \Delta(z, w) \mathbf{G}(z, w)=\overleftarrow{q}(z, w) \\
& C q(z, w)+D \Delta(z, w) \mathbf{G}(z, w)=\mathbf{G}(z, w)
\end{aligned}
$$

The latter formula implies

$$
\mathbf{G}(z, w)=q(z, w)(I-D \Delta(z, w))^{-1} C,
$$

and in turn the former formula implies

$$
A+B \Delta(z, w)(I-D \Delta(z, w))^{-1} C=\frac{\overleftarrow{q}(z, w)}{q(z, w)}
$$

Since $\overleftarrow{q} / q$ is already in reduced terms we must have $d_{1} \geq n$ and $d_{2} \geq m$. We already know $d_{1} \leq n$ and $d_{2} \leq m$ (see Proposition 5.3). Therefore, $n=\operatorname{dim} \Theta_{\mu}$ and $m=\operatorname{dim} \mathbb{\Psi}_{\mu}$, and the result follows.

Theorem 6.8 (spectral matching). Let $\mu$ and $\rho$ be two OC measures. Suppose $\operatorname{Max}_{\mu}=\operatorname{Max}_{\rho} \neq\{0\}$ and let $q \in \operatorname{Max}_{\mu}$. Assume $q$ and $\overleftarrow{q}$ have no common factor. Then, $\square_{\mu}=\square_{\rho}$ and the inner products $\langle\cdot, \cdot\rangle_{\mu}$ and $\langle\cdot, \cdot\rangle_{\rho}$ agree up to a constant multiple on $\square_{\mu}$; that is,

$$
\frac{1}{\|q\|_{L^{2}(\mu)}^{2}}\langle f, g\rangle_{\mu}=\frac{1}{\|q\|_{L^{2}(\rho)}^{2}}\langle f, g\rangle_{\rho} \quad \text { for all } f, g \in \square_{\mu} .
$$

In other words,

$$
\frac{1}{\|q\|_{L^{2}(\mu)}^{2}} K \square_{\mu}=\frac{1}{\|q\|_{L^{2}(\rho)}^{2}} K \square_{\rho}
$$


Proof. We may renormalize $\mu$ and $\rho$ so that $1=\|q\|_{L^{2}(\mu)}=\|q\|_{L^{2}(\rho)}$.

By choosing orthonormal bases for the $n$-dimensional subspaces (by Lemma 6.7) $\Theta_{\mu}$ and $\Theta_{\rho}$, we may write

$$
\begin{aligned}
K \Theta_{\mu}((z, w),(Z, W)) & =\left\langle\mathbf{E}_{\mu}(z, w), \mathbf{E}_{\mu}(Z, W)\right\rangle, \\
K \Theta_{\rho}((z, w),(Z, W)) & =\left\langle\mathbf{E}_{\rho}(z, w), \mathbf{E}_{\rho}(Z, W)\right\rangle,
\end{aligned}
$$

for $\mathbf{E}_{\mu}, \mathbf{E}_{\rho} \in \mathbb{C}^{n}[z, w]$.

Likewise, we may write the $m$-dimensional subspaces $\square_{\mu}$ and $\Psi_{\rho}$ as

$$
\begin{aligned}
& K \mathbb{\boxplus}_{\mu}((z, w),(Z, W))=\left\langle\mathbf{F}_{\mu}(z, w), \mathbf{F}_{\mu}(Z, W)\right\rangle, \\
& K \mathbb{\boxplus}_{\rho}((z, w),(Z, W))=\left\langle\mathbf{F}_{\rho}(z, w), \mathbf{F}_{\rho}(Z, W)\right\rangle,
\end{aligned}
$$

where $\mathbf{F}_{\mu}, \mathbf{F}_{\rho} \in \mathbb{C}^{m}[z, w]$.

By Proposition 5.3, both $\mathbf{E}_{\mu}, \mathbf{F}_{\mu}$ and $\mathbf{E}_{\rho}, \mathbf{F}_{\rho}$ satisfy the hypotheses of Lemma 3.4 (in place of $\mathbf{E}, \mathbf{F}$ and $\tilde{\mathbf{E}}, \tilde{\mathbf{F}})$, since by Theorem 6.1, we have

$$
\begin{aligned}
(1-z \bar{Z}) K \mathbf{\Xi}_{\mu}((z, w),(Z, W))+ & (1-w \bar{W}) K \Psi_{\mu}((z, w),(Z, W)) \\
= & (1-z \bar{Z}) K \Theta_{\rho}((z, w),(Z, W))+(1-w \bar{W}) K \mathbb{\Psi}_{\rho}((z, w),(Z, W)) .
\end{aligned}
$$

Therefore, $\mathbf{E}_{\mu}$ is a unitary multiple of $\mathbf{E}_{\rho}$ and $\mathbf{F}_{\mu}$ is a unitary multiple of $\mathbf{F}_{\rho}$. In other words,

$$
\begin{aligned}
& K \Psi_{\mu}((z, w),(Z, W))=K \varpi_{\rho}((z, w),(Z, W)), \\
& K \Xi_{\mu}((z, w),(Z, W))=K \Xi_{\rho}((z, w),(Z, W)) .
\end{aligned}
$$

Now we will see that this is all that is needed to reassemble the two inner products on $\square_{\mu}$ or $\square_{\rho}$.

By reflection

$$
K \mathbb{\Xi}_{\mu}((z, w),(Z, W))=K \mathbb{\varpi}_{\rho}((z, w),(Z, W)),
$$

and by the formulas (which hold for both $\mu$ and $\rho$ )

$$
K \mathbb{\varpi}_{\mu}-K \mathbb{\boxplus}_{\mu}=\left(1-|z|^{2}\right) K \boldsymbol{\square}_{\mu} \quad \text { (Proposition 5.5) }
$$

and

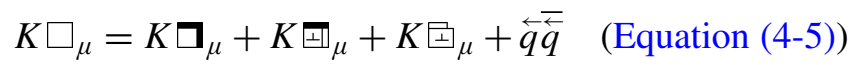

where every reproducing kernel is evaluated on the diagonal $(z, w)=(Z, W)$, we see that

$$
K \square_{\mu}=K \square_{\rho} .
$$

(This is similar to the argument in the proof of Proposition 6.3.)

\section{Bernstein-Szegó measures}

Converse to the previous section, we now study Bernstein-Szegó measures, which will be shown to be OC measures. Bernstein-Szegó measures are measures on $\mathbb{T}^{2}$ of the form

$$
d \mu=\frac{1}{|q(z, w)|^{2}} d \sigma(z, w),
$$


where $q \in \mathbb{C}[z, w]$ has no zeros on $\mathbb{D}^{2}$. (Recall $d \sigma$ is normalized Lebesgue measure on $\mathbb{T}^{2}$.)

The following proposition looks innocuous, but it addresses the main technical difficulty not present in the case of polynomials with no zeros on the entire closed bidisk. Note this proposition does not require the polynomial to have finitely many zeros on $\mathbb{T}^{2}$.

Proposition 7.1. Let $q \in \mathbb{C}[z, w]$ have degree at most $(n, m)$ and no zeros on $\mathbb{D}^{2}$. Define a measure on $\mathbb{T}^{2}$ by

$$
d \mu=\frac{1}{|q(z, w)|^{2}} d \sigma(z, w) .
$$

Then, $q \in \operatorname{Max}_{\mu}$ and more generally

$$
q \perp_{\mu}\left\{f \in L^{2}(\mu): \hat{f}(j, k)=0 \text { for } k<0 \text { and for } k=0 \text { and } j \leq 0\right\} .
$$

Proof. Let $f \in L^{2}(\mu)$ satisfy

$$
\hat{f}(j, k)=0 \quad \text { for } k<0 \text { and for } k=0 \text { and } j \leq 0 .
$$

It is necessarily true that $f \in L^{2}\left(\mathbb{T}^{2}\right)$. For almost every $z \in \mathbb{T}$, the function $f_{(z)}(w)=f(z, w)$ is in $L^{2}(\mathbb{T})$ and since $\hat{f}(j, k)=0$ for $k<0, f_{(z)}$ is actually in $H^{2}(\mathbb{T})$ for almost every $z \in \mathbb{T}$.

So, the function (of $w$ )

$$
g_{(z)}(w):=\frac{f(z, w)}{q(z, w)}
$$

is in the Smirnov class $N^{+}$(which consists of all ratios of bounded analytic functions with outer denominator; see [Duren 1970, Section 2.5]), for almost every $z \in \mathbb{T}: q(z, \cdot)$ has no zeros in the disk for all but finitely many $z \in \mathbb{T}$ (by Lemma 3.5) and is therefore outer for almost every $z \in \mathbb{T}$. Since $f \in L^{2}(\mu)$, Fubini's theorem says that for almost every $z \in \mathbb{T}$, we have $g_{(z)} \in L^{2}(\mathbb{T})$. By Theorem 2.11 in [Duren $1970], N^{+} \cap L^{2}(\mathbb{T})=H^{2}(\mathbb{T})$, and therefore $g_{(z)} \in H^{2}(\mathbb{T})$ for almost every $z \in \mathbb{T}$.

Owing to the fact that $g_{(z)}$ is orthogonal to $w^{j}$ for $j<0$,

$$
f(z, 0)=\int_{\mathbb{T}} f(z, w) \frac{d w}{2 \pi i w}=\int_{\mathbb{T}} \frac{f(z, w)}{q(z, w)} q(z, w) \frac{d w}{2 \pi i w}=\int_{\mathbb{T}} \frac{f(z, w)}{q(z, w)} q(z, 0) \frac{d w}{2 \pi i w}
$$

for almost every $z \in \mathbb{T}$, and so

$$
\int_{\mathbb{T}^{2}} \frac{f(z, w)}{q(z, w)} \frac{d w}{2 \pi i w} \frac{d z}{2 \pi i z}=\int_{\mathbb{T}} \frac{f(z, 0)}{q(z, 0)} \frac{d z}{2 \pi i z} .
$$

Now, the function defined by $h(z)=f(z, 0) / q(z, 0)$ is in $L^{2}(\mathbb{T})$ by Fubini's theorem. Also, $h$ is in the Smirnov class $N^{+}$because $f(\cdot, 0)$ is in $H^{2}(\mathbb{T})$ (by the assumption that $\hat{f}(j, 0)=0$ for $j \leq 0$ ), and $q(\cdot, 0)$ is outer since $q(z, 0)$ has no zeros in the disk. Therefore, $h$ is in $H^{2}(\mathbb{T})$. Thus, we may conclude

$$
\int_{\mathbb{T}^{2}} \frac{f(z, w)}{q(z, w)} \frac{d w}{2 \pi i w} \frac{d z}{2 \pi i z}=\int_{\mathbb{T}} \frac{f(z, 0)}{q(z, 0)} \frac{d z}{2 \pi i z}=\frac{f(0,0)}{q(0,0)}=0,
$$

since $\hat{f}(0,0)=0$. 
Since

$$
\langle f, q\rangle_{\mu}=\int_{\mathbb{T}^{2}} \frac{f(z, w) \overline{q(z, w)}}{|q(z, w)|^{2}} d \sigma(z, w)=\int_{\mathbb{T}^{2}} \frac{f(z, w)}{q(z, w)} d \sigma(z, w),
$$

we have shown $\langle f, q\rangle_{\mu}=0$, or in other words $f \perp_{\mu} q$.

From here, the proofs follow the stable case, as in [Knese 2008], with some minor changes.

Corollary 7.2. If $f \in L^{2}(\mu) \cap H^{2}\left(\mathbb{T}^{2}\right)$ and

$$
\hat{f}(j, k)=0 \text { for } k>m \text { and for } k=m \text { and } j \geq n,
$$

then $\langle f, \overleftarrow{q} g\rangle_{\mu}=0$ for any $g \in H^{\infty}\left(\mathbb{\mathbb { T }}^{2}\right)$

Proof. Notice that $\langle\overleftarrow{q} g, f\rangle_{\mu}=\left\langle\bar{f} g z^{n} w^{m}, q\right\rangle_{\mu}$. Also, notice that $\bar{f} g z^{n} w^{m}$ satisfies the hypotheses of the previous proposition (it helps to draw a picture of the frequency support of $f$ and $\bar{f} g z^{n} w^{m}$ ). Therefore, $\langle f, \overleftarrow{q} g\rangle_{\mu}=0$

\section{Lemma 7.3. Define}

$$
L_{(Z, W)}(z, w)=L((z, w),(Z, W))=(z \bar{Z})^{n} \frac{q(z, w) \overline{q(1 / \bar{z}, W)}-\overleftarrow{q}(z, w) \bar{q}(1 / \bar{z}, W)}{(1-z \bar{Z})(1-w \bar{W})}
$$

Suppose $f \in L^{2}(\mu) \cap H^{2}\left(\mathbb{T}^{2}\right)$, with $\hat{f}(j, k)=0$ for $k>m$ and for $k=m$ and $j \geq n$. Then, for $(Z, W) \in \mathbb{D}^{2}$,

$$
\sum_{k=0}^{m-1} \sum_{j=n}^{\infty} \hat{f}(j, k) Z^{j} W^{k}=\left\langle f, L_{(Z, W)}\right\rangle_{\mu}
$$

Proof. By Corollary 7.2, $f$ is orthogonal to the function

$$
G_{(Z, W)}(z, w)=\frac{\overleftarrow{q}(z, w) z^{n} \bar{q}(1 / \bar{z}, W)}{(1-z \bar{Z})(1-w \bar{W})}
$$

for each $(Z, W) \in \mathbb{D}^{2}$.

Therefore,

$$
\begin{aligned}
\left\langle f, L_{(Z, W)}\right\rangle_{\mu} & =\int_{\mathbb{T}^{2}} \frac{f(z, w) \overline{q(z, w)} q(z, W)(\bar{z} Z)^{n}}{(1-\bar{z} Z)(1-\bar{w} W)|q(z, w)|^{2}} \frac{d w d z}{(2 \pi i)^{2} z w} \\
& =\int_{\mathbb{T}} \int_{\mathbb{T}} \frac{f(z, w) q(z, W)(\bar{z} Z)^{n}}{(1-\bar{z} Z)(w-W) q(z, w)} \frac{d w}{2 \pi i} \frac{d z}{2 \pi i z} \\
& =\int_{\mathbb{T}} \frac{f(z, W)}{q(z, W)} q(z, W) \frac{(\bar{z} Z)^{n}}{(1-\bar{z} Z)} \frac{d z}{2 \pi i z} \\
& =\sum_{j=n}^{\infty} \sum_{k=0}^{m-1} \hat{f}(j, k) Z^{j} W^{k} .
\end{aligned}
$$

Going from (7-2) to (7-3) is an application of the Cauchy integral formula and going from (7-3) to (7-4) involves cancellation and another application of the Cauchy integral formula. 
Theorem 7.4. Let $q$ be a nonzero polynomial of degree at most $(n, m)$ with no zeros on $\mathbb{D}^{2}$. Define a measure on $\mathbb{T}^{2}$ by

$$
d \mu=\frac{1}{|q(z, w)|^{2}} d \sigma(z, w)
$$

Then, $\mu$ is an OC measure.

Proof. Let

$$
\mathrm{HS}=\left\{f \in L^{2}(\mu) \cap H^{2}\left(\mathbb{T}^{2}\right): \hat{f}(j, k)=0 \text { for } k \geq m\right\},
$$

$(\mathrm{HS}=$ half strip $)$ and let

$$
\mathrm{NHS}=\left\{f \in L^{2}(\mu) \cap H^{2}\left(\mathbb{T}^{2}\right): \hat{f}(j, k)=0 \quad \text { for } k>m \text { and when } k=m \text { and } j \geq n\right\},
$$

(NHS $=$ notched half strip) .

We claim that NHS $\ominus_{\mu} \mathrm{HS}=\mathbf{\theta}_{\mu}$. To prove NHS $\ominus_{\mu} \mathrm{HS} \subset \mathbf{\theta}_{\mu}$, notice that $L_{(Z, W)}$ from Lemma 7.3 is in HS since the numerator of $L_{(Z, W)}$ vanishes when $w=1 / \bar{W}$, and hence $L_{(Z, W)}$ is a polynomial of degree at most $m-1$ in $w$. So, if $f \in \mathrm{NHS} \ominus_{\mu} \mathrm{HS}$, then

$$
0=\left\langle f, L_{(Z, W)}\right\rangle_{\mu}=\sum_{j=n}^{\infty} \sum_{k=0}^{m-1} \hat{f}(j, k) Z^{j} W^{k},
$$

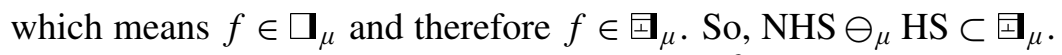

To prove that $\mathbb{\Theta}_{\mu} \subset$ NHS $\ominus_{\mu}$ HS, let $P_{\mathrm{HS}}: L^{2}(\mu) \rightarrow$ HS denote the orthogonal projection onto HS, a necessarily closed subspace of $L^{2}(\mu)$ (the topology on $L^{2}(\mu)$ is finer than the topology on $L^{2}\left(\mathbb{T}^{2}\right)$ ). If $f \in \mathbb{\Xi}_{\mu}$ then

$$
f-P_{\mathrm{HS}} f \in \mathrm{NHS} \ominus_{\mu} \mathrm{HS} \subset \text { 田 }_{\mu},
$$

and this implies

$$
P_{\mathrm{HS}} f \in \text { 田 }_{\mu} \cap \mathrm{HS}=\{0\} .
$$

Hence, $P_{\mathrm{HS}} f=0$ which means $f \perp_{\mu}$ HS. In other words, $f \in \mathrm{NHS} \ominus_{\mu}$ HS. Hence, NHS $\ominus_{\mu} \mathrm{HS}=\mathbf{\theta}_{\mu}$.

Now, since $\mathbf{\Xi}_{\mu} \subset \mathrm{NHS} \ominus_{\mu} \mathrm{HS}$, it follows that $\boldsymbol{\Xi}_{\mu} \subset \mathrm{\Xi}_{\mu}$. A similar argument to the above (using the projection $P_{\mathrm{HS}}$ ) proves $\Xi_{\mu} \subset \mathrm{NHS} \ominus_{\mu} \mathrm{HS}=\mathbf{\theta}_{\mu}$. This implies $\mathbf{\Xi}_{\mu}=\Xi_{\mu}$; namely, $\mu$ is an OC measure.

Corollary 7.5. Let $q$ be a nonzero polynomial of degree at most $(n, m)$ with no zeros on $\mathbb{D}^{2}$. Define a measure on $\mathbb{T}^{2}$ by

$$
d \mu=\frac{1}{|q(z, w)|^{2}} d \sigma(z, w)
$$

Then,

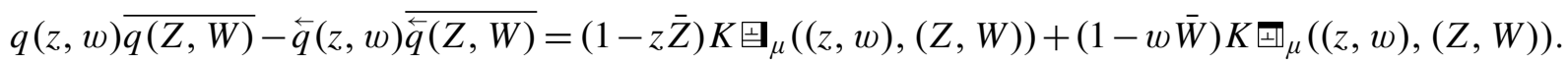

Proof. Proposition 7.1 says $q \in \operatorname{Max}_{\mu}$ and Theorem 7.4 says $\mathbb{\Xi}_{\mu}=\mathbb{\Xi}_{\mu}$. Since $\|q\|_{L^{2}(\mu)}=1$, the conclusion follows from Theorem 6.1 since $\Xi_{\mu}=\mathbb{\Xi}_{\mu}$ says $\epsilon=0$. 
Corollary 7.6 ("Bernstein-Szegő approximation"). Let $\rho$ be an OC measure and suppose $q \in \operatorname{Max}_{\rho}$ has no factors in common with $\overleftarrow{q}$. Define

$$
d \mu=\frac{1}{|q(z, w)|^{2}} d \sigma(z, w) .
$$

If we normalize $\rho$ so that $\|q\|_{L^{2}(\rho)}=1$, then $\square_{\rho}=\square_{\mu}$ and

$$
K \square_{\rho}=K \square_{\mu},
$$

that is, the inner products on $\square_{\mu}$ and $\square_{\rho}$ from $L^{2}(\mu)$ and $L^{2}(\rho)$ agree.

Proof. By Proposition 7.1 $q \in \operatorname{Max}_{\mu}$ and by Theorem 7.4, $\mu$ is an OC measure. We have assumed $q$ has no factors in common with $\overleftarrow{q}$ and this allows us to apply Theorem 6.8, from which the conclusion follows immediately.

One final lemma will make the proof of the main theorem a matter of bookkeeping. We use the following notations:

$$
\begin{aligned}
Z_{q} & =\left\{(z, w) \in \mathbb{C}^{2}: q(z, w)=0\right\}, \\
\pi_{1}(z, w) & =z \text { and } \pi_{2}(z, w)=w .
\end{aligned}
$$

Lemma 7.7. If $\mu$ is the Bernstein-Szegó measure associated to $q \in \mathbb{C}[z, w]$, that is,

$$
d \mu=\frac{1}{|q(z, w)|^{2}} d \sigma(z, w),
$$

then $J(z, w)=\left(w-w_{0}\right)$ and $L(z, w)=\left(z-z_{0}\right)$ will be divisors of the ideal $\Phi_{\mu}$ whenever $w_{0} \notin \pi_{2}\left(Z_{q} \cap \mathbb{T}^{2}\right)$ and $z_{0} \notin \pi_{1}\left(Z_{q} \cap \mathbb{T}^{2}\right)$, respectively.

Proof. If $\left(z-z_{0}\right) f(z, w) \in L^{2}(\mu)$ for some $f \in \mathbb{C}[z, w]$ and $z_{0} \notin \pi_{1}\left(Z_{q} \cap \mathbb{T}^{2}\right)$, then let $U$ be a neighborhood of $Z_{z-z_{0}} \cap \mathbb{T}^{2}$ which does not intersect $Z_{q}$. Then, $\left|z-z_{0}\right|^{2}$ is bounded below on $\mathbb{T}^{2} \backslash U$ and $|q|^{2}$ is bounded below on $U$, say by a constant $c$. Then,

$$
\infty>\int_{\mathbb{T}^{2}} \frac{\left|z-z_{0}\right|^{2}|f(z, w)|^{2}}{|q(z, w)|^{2}} d \sigma \geq \int_{\mathbb{T}^{2} \backslash U} \frac{c|f(z, w)|^{2}}{|q(z, w)|^{2}} d \sigma
$$

and

$$
\infty>\int_{U}|f(z, w)|^{2} d \sigma \geq \int_{U} \frac{c|f(z, w)|^{2}}{|q(z, w)|^{2}} d \sigma
$$

together imply

$$
\|f\|_{L^{2}(\mu)}^{2}=\int_{U} \frac{|f(z, w)|^{2}}{|q(z, w)|^{2}} d \sigma+\int_{\mathbb{T}^{2} \backslash U} \frac{|f(z, w)|^{2}}{|q(z, w)|^{2}} d \sigma<\infty .
$$

So, $L$ is a divisor of $\Phi_{\mu}$. The proof for $J$ is similar. 


\section{Proof of the main theorem}

We have all of the pieces in place to prove the theorem from the introduction. Here is the main theorem with extra details filled in. When we use the inner product notation $\langle\cdot, \cdot\rangle$ below with no subscript, we are taking inner products in $\mathbb{C}^{N}$ (where the $N$ is taken from context) and not taking any kind of Hilbert function space inner product.

Theorem 8.1. Let $q \in \mathbb{C}[z, w]$ be almost stable with $\operatorname{deg} q \leq(n, m)$. Then, there exist vector polynomials $\mathbf{E} \in \mathbb{C}^{n}[z, w]$ and $\mathbf{F} \in \mathbb{C}^{m}[z, w]$, with $\operatorname{deg} \mathbf{E} \leq(n-1, m)$, and $\operatorname{deg} \mathbf{F} \leq(n, m-1)$, satisfying the following conditions:

(1) $\mathbf{E}$ is horizontally $\mathbb{D} \cup X$-stable where $X=\mathbb{T} \backslash\left(\pi_{2}\left(Z_{q}\right)\right)$.

(2) $\overleftarrow{\mathbf{F}}$ is vertically $\mathbb{D} \cup Y$-stable where $Y=\mathbb{T} \backslash\left(\pi_{1}\left(Z_{q}\right)\right)$

(3) $q(z, w) \overline{q(Z, W)}-\overleftarrow{q}(z, w) \bar{q}(Z, W)$

$$
=(1-z \bar{Z})\langle\mathbf{E}(z, w), \mathbf{E}(Z, W)\rangle+(1-w \bar{W})\langle\mathbf{F}(z, w), \mathbf{F}(Z, W)\rangle .
$$

(4) If $\tilde{\mathbf{E}} \in \mathbb{C}^{n}[z, w]$ and $\tilde{\mathbf{F}} \in \mathbb{C}^{m}[z, w]$ satisfy items (1) and (3) above in place of $\mathbf{E}$ and $\mathbf{F}$, then there exist unitary matrices $U_{1}, U_{2}$ such that

$$
\mathbf{E}(z, w)=U_{1} \tilde{\mathbf{E}}(z, w) \quad \text { and } \quad \mathbf{F}(z, w)=U_{2} \tilde{\mathbf{F}}(z, w) .
$$

Proof. We use the setup (and conclusion) of Corollary 7.5. By Lemma 6.7, 目 $\mu$ has dimension $n$ and $\mathbf{I}_{\mu}$ has dimension $m$. Let $\left\{e_{1}, \ldots, e_{n}\right\}$ be an orthonormal basis of $\boldsymbol{\theta}_{\mu}$ and $\left\{f_{1}, \ldots, f_{m}\right\}$ an orthonormal basis of $\mathbf{I}_{\mu}$. Define $\mathbf{E}=\left(e_{1}, \ldots, e_{n}\right)^{t} \in \mathbb{C}^{n}[z, w]$ and $\mathbf{F}=\left(f_{1}, \ldots, f_{m}\right)^{t} \in \mathbb{C}^{m}[z, w]$. Corollary 7.5 now proves item (3).

Write $\mathbf{E}(z, w)=E(w) \Lambda_{n}(z)$ and $\mathbf{F}(z, w)=F(z) \Lambda_{m}(w)$. With these choices, Proposition 5.3 says $E(w)$ is invertible for all $w \in \overline{\mathbb{D}}$ with the exception of $w_{0} \in \mathbb{\mathbb { V }}$ with the property that $w-w_{0}$ is not a divisor of $\Phi_{\mu}$. Lemma 7.7 says $\left(w-w_{0}\right)$ is a divisor of $\Phi_{\mu}$ when $w_{0} \notin \pi_{2}\left(Z_{q} \cap \mathbb{T}^{2}\right)$. So, $E(w)$ is invertible when $w \in \overline{\mathbb{D}} \backslash \pi_{2}\left(Z_{q} \cap \mathbb{\mathbb { T }}^{2}\right)$. The entries of

$$
\overleftarrow{\mathbf{F}}(z, w)=z^{n} w^{m-1} \overline{\mathbf{F}(1 / \bar{z}, 1 / \bar{w})}
$$

form an orthonormal basis for $\mathbb{\Psi}_{\mu}$ and

$$
\overleftarrow{\mathbf{F}}(z, w)=z^{n} \overline{F(1 / \bar{z})} w^{m-1} \overline{\Lambda_{m}(1 / \bar{w})}=z^{n} \overline{F(1 / \bar{z})} \chi \Lambda_{m}(w)
$$

where $\chi$ is the $m \times m$ matrix with ones on the antidiagonal (entries $(j, m-j)$ ) and zeros elsewhere. By Proposition 5.3 and Lemma $7.7 z^{n} \overline{F(1 / \bar{z})} \chi$ is invertible for $z \in \overline{\mathbb{D}} \backslash \pi_{1}\left(Z_{q} \cap \mathbb{T}^{2}\right)$. Of course, $\chi$ is invertible, so the same statement holds for $z^{n} \overline{F(1 / \bar{z})}$. This proves items (1) and (2) of Theorem 8.1.

Lemma 3.4 proves item (4).

\section{Polynomials with unique decompositions}

In this section we give a characterization of the polynomials with no zeros on the bidisk that have a unique sums of squares decomposition. 
Proof of Theorem 1.15. Suppose $q$ is almost stable with $\operatorname{deg} p=(n, m)$.

To prove item (1) implies (2) in Theorem 1.15, suppose there are unique $\Gamma_{1}$ and $\Gamma_{2}$, sums of squared moduli of two-variable polynomials, such that

$$
|q(z, w)|^{2}-|\overleftarrow{q}(z, w)|^{2}=\left(1-|z|^{2}\right) \Gamma_{1}(z, w)+\left(1-|w|^{2}\right) \Gamma_{2}(z, w) .
$$

By Corollary 7.5, if $\mu$ is the Bernstein-Szegő measure associated to $q$ then

$$
\begin{aligned}
|q(z, w)|^{2}-|\overleftarrow{q}(z, w)|^{2} & =\left(1-|z|^{2}\right) K \mathbf{\Xi}_{\mu}((z, w),(z, w))+\left(1-|w|^{2}\right) K \varpi_{\mu}((z, w),(z, w)) \\
& =\left(1-|z|^{2}\right) K \text { 田 }_{\mu}((z, w),(z, w))+\left(1-|w|^{2}\right) K \mathbb{\boxplus}_{\mu}((z, w),(z, w)) .
\end{aligned}
$$

These reproducing kernels can be written as sums of squares of two variable polynomials. Since we are assuming such decompositions are unique we have

$$
K \text { 四 }_{\mu}((z, w),(z, w))=K \text { 田 }_{\mu}((z, w),(z, w)) .
$$

Because of the formula (Proposition 5.5)

$$
K \Theta_{\mu}((z, w),(z, w))-K \text { 田 }_{\mu}((z, w),(z, w))=\left(1-|w|^{2}\right) K \mathbf{\square}_{\mu}((z, w),(z, w)),
$$

we see that

$$
K \boldsymbol{\square}_{\mu}((z, w),(z, w))=0 .
$$

This implies $\boldsymbol{\square}_{\mu}=\{0\}$. In other words, there are no nonzero $f \in \boldsymbol{\square} \cap L^{2}(\mu)=\boldsymbol{\square} \cap L^{2}\left(1 /|q|^{2} d \sigma\right)$ and this just says there are no nonzero $f \in \boldsymbol{\square}$ such that

$$
f / q \in L^{2}\left(\mathbb{T}^{2}\right) .
$$

This proves that item (1) implies item (2) in Theorem 1.15.

To prove item (2) implies (3) in the theorem, assume there are no nonzero $f \in \boldsymbol{\square}$ such that

$$
f / q \in L^{2}\left(\mathbb{T}^{2}\right) .
$$

Notationally, $\boldsymbol{\square}_{\mu}=\{0\}$ and again by (9-1) we have

$$
K \text { 四 }_{\mu}((z, w),(z, w))=K \text { 田 }_{\mu}((z, w),(z, w)) .
$$

The two subspaces $\Theta_{\mu}$ and $\Theta_{\mu}$ are reflections of one another. So, if we write

$$
K \text { 目 }_{\mu}((z, w),(z, w))=K \text { 田 }_{\mu}((z, w),(z, w))=|\mathbf{E}(z, w)|^{2},
$$

where $\mathbf{E}(z, w)=\left(E_{1}(z, w), \ldots, E_{n}(z, w)\right)^{t} \in \mathbb{C}^{n}[z, w]$ and $E_{1}, \ldots, E_{n}$ are an orthonormal basis for $\boldsymbol{\Theta}_{\mu}=\mathbf{\Xi}_{\mu}$, then the entries of

$$
\overleftarrow{\mathbf{E}}(z, w):=z^{n-1} w^{m} \overline{\mathbf{E}(1 / \bar{z}, 1 / \bar{w})}
$$

also form an orthonormal basis for $\Theta_{\mu}=\Theta_{\mu}$. So,

$$
|\mathbf{E}(z, w)|^{2}=|\overleftarrow{\mathbf{E}}(z, w)|^{2},
$$

and by Lemma 3.2 there is an $n \times n$ unitary matrix $U$ such that

$$
U \mathbf{E}(z, w)=\overleftarrow{\mathbf{E}}(z, w)
$$


(As we commented there Lemma 3.2 holds for two-variable polynomials just as well.) If we reflect both sides of this equation (take conjugates, replace $(z, w)$ with $(1 / \bar{z}, 1 / \bar{w})$, and multiply through by $\left.z^{n-1} w^{m}\right)$ we see that

$$
\bar{U} \overleftarrow{\mathbf{E}}(z, w)=\mathbf{E}(z, w)
$$

Note that $\bar{U}$ is the matrix obtained by taking complex conjugates of each entry of $U$ and is not the adjoint of $U$. In fact, $\bar{U}^{-1}=U^{t}$ and therefore

$$
U^{t} \mathbf{E}(z, w)=\overleftarrow{\mathbf{E}}(z, w)=U \mathbf{E}(z, w)
$$

Hence, $U=U^{t}$ since the vectors $\mathbf{E}(z, w)$ span all of $\mathbb{C}^{n}$ as $(z, w)$ varies over $\mathbb{C}^{2}$ (by Proposition 5.3). The matrix $U$ is therefore symmetric unitary. Symmetric unitaries can be factored as $U=V^{t} V$ where $V$ is a unitary — this is the so-called Takagi factorization. The vector polynomial

$$
V \mathbf{E}(z, w)
$$

is then symmetric since its reflection is

$$
\bar{V} \overleftarrow{\mathbf{E}}(z, w)=\left(V^{t}\right)^{-1} U \mathbf{E}(z, w)=V \mathbf{E}(z, w)
$$

as $U=V^{t} V$. So we replace $\mathbf{E}$ with $V \mathbf{E}$ and this proves there exists a symmetric vector polynomial $\mathbf{E}$ such that

$$
K \Theta_{\mu}((z, w),(z, w))=K \text { 田 }_{\mu}((z, w),(z, w))=|\mathbf{E}(z, w)|^{2} .
$$

By Proposition 5.3, E is horizontally $\mathbb{D} \cup \mathbb{E}$-stable, since $\mathbf{E}$ and $\overleftarrow{\mathbf{E}}$ are both horizontally $\mathbb{D}$-stable.

Similar arguments show that when $\mathbf{\square}_{\mu}=\{0\}$, there exists a symmetric vector polynomial $\mathbf{F} \in \mathbb{C}^{m}[z, w]$ of degree $(n, m-1)$ which is vertically $\mathbb{D} \cup \mathbb{E}$-stable, and

$$
K \mathbb{\Psi}_{\mu}((z, w),(z, w))=K \mathbb{\Psi}_{\mu}((z, w),(z, w))=|\mathbf{F}(z, w)|^{2} .
$$

By Corollary 7.5, we have that

$$
|q(z, w)|^{2}-|\overleftarrow{q}(z, w)|^{2}=\left(1-|z|^{2}\right)|\mathbf{E}(z, w)|^{2}+\left(1-|w|^{2}\right)|\mathbf{F}(z, w)|^{2}
$$

where $\mathbf{E}$ and $\mathbf{F}$ satisfy all of the desired properties. This proves item (2) implies item (3).

To prove item (3) implies (1) assume (9-2) holds where $\mathbf{E}$ is horizontally $\mathbb{D}$-stable and $\mathbf{F}$ is vertically $\mathbb{D}$-stable. We must show this is the only sums of squares decomposition for $q$.

Suppose there are vector polynomials $\mathbf{A} \in \mathbb{C}^{N}[z, w], \mathbf{B} \in \mathbb{C}^{M}[z, w]$ such that

$$
|q(z, w)|^{2}-|\overleftarrow{q}(z, w)|^{2}=\left(1-|z|^{2}\right)|\mathbf{A}(z, w)|^{2}+\left(1-|w|^{2}\right)|\mathbf{B}(z, w)|^{2}
$$

Setting $|w|=1$, Equation (9-2) implies

$$
|\mathbf{E}(z, w)|^{2}=|\mathbf{A}(z, w)|^{2} \quad \text { for }(z, w) \in \mathbb{C} \times \mathbb{T} .
$$

Since $\mathbf{E}$ is horizontally $\mathbb{D}$-stable, Lemma 3.3 applies: $n \leq N$ and there exists a one variable $N \times n$ matrix valued rational inner function $\Psi_{1}$ such that

$$
\mathbf{A}(z, w)=\Psi_{1}(w) \mathbf{E}(z, w) \quad \text { for }(z, w) \in \mathbb{D}^{2} .
$$


By similar reasoning, $m \leq M$ and there exists an $M \times m$ matrix valued rational inner function $\Psi_{2}$ such that

$$
\mathbf{B}(z, w)=\Psi_{2}(z) \mathbf{F}(z, w) .
$$

So,

$$
|\mathbf{A}(z, w)|^{2} \leq|\mathbf{E}(z, w)|^{2}, \quad|\mathbf{B}(z, w)|^{2} \leq|\mathbf{F}(z, w)|^{2} \quad \text { for all }(z, w) \in \mathbb{D}^{2} .
$$

However, we must have equality at every point in both of these inequalities because otherwise

$$
\left(1-|z|^{2}\right)|\mathbf{E}(z, w)|^{2}+\left(1-|w|^{2}\right)|\mathbf{F}(z, w)|^{2}=\left(1-|z|^{2}\right)|\mathbf{A}(z, w)|^{2}+\left(1-|w|^{2}\right)|\mathbf{B}(z, w)|^{2}
$$

would be violated. Hence, the sums of squares terms for $q$ are unique:

$$
|\mathbf{A}(z, w)|^{2}=|\mathbf{E}(z, w)|^{2}, \quad|\mathbf{B}(z, w)|^{2}=|\mathbf{F}(z, w)|^{2} \quad \text { for all }(z, w) \in \mathbb{C}^{2} .
$$

This proves (3) implies (1) and concludes the proof.

Corollary 1.16 says that the only stable polynomials with a unique decomposition are one variable polynomials. We prove this now.

Proof of Corollary 1.16. Suppose $p \in \mathbb{C}[z, w]$ is stable and deg $p=(n, m)$. It is implicit in most of this paper that $n, m>0$. By Theorem 1.15 , since $1 /|p|^{2}$ is integrable, it follows that $p$ does not have a unique sums of squares decomposition. If $n=0$ or $m=0$ then $p$ is really just a one variable polynomial with no zeros on closed disk. It is well known that the decomposition in the one variable Christoffel-Darboux formula is unique, since the sums of squares term can just be solved for; it equals

$$
\frac{|p(z)|^{2}-|\overleftarrow{p}(z)|^{2}}{1-|z|^{2}}
$$

in the case where $m=0$.

\section{Application: Fejér-Riesz factorization}

The classical Fejér-Riesz theorem says that a nonnegative one variable trigonometric polynomial $t$ can be factored as $|p(z)|^{2}$ where $p \in \mathbb{C}[z]$ has no zeros in the disk $\mathbb{D}$. It is false that all nonnegative two variable trig polynomials can be factored as $|p(z, w)|^{2}$ where $p \in \mathbb{C}[z, w]$ has no zeros on the bidisk. Indeed, Geronimo and Woerdeman [2004] give a characterization of which strictly positive trig polynomials have a "Fejér-Riesz type factorization". We reprove and extend this result to certain cases of nonnegative trigonometric polynomials. Our proof does not make use of a certain "maximal entropy result" and is therefore self-contained.

We emphasize that requiring a finite measure $\mu$ to be an OC measure is a condition on its moments [Knese 2008, Appendix]. First, let us establish the strictly positive result.

Theorem 10.1 [Geronimo and Woerdeman 2004]. Let $t: \mathbb{T}^{2} \rightarrow \mathbb{C}$ be a positive trigonometric polynomial of two variables with Fourier coefficients $\hat{t}(j, k)$ supported on the set $|j| \leq n,|k| \leq m$. Then, there exists a stable $p \in \mathbb{C}[z, w]$, deg $p \leq(n, m)$ satisfying $t(z, w)=|p(z, w)|^{2}$ for all $(z, w) \in \mathbb{T}^{2}$ if and only if the measure $d \mu=(1 / t) d \sigma$ is an OC measure. 
Proof. The "only if" direction follows from Theorem 7.4. To prove the "if" direction, observe that if $\mu$ is an OC measure, then by Corollary 7.6, if $p$ is a unit norm polynomial in $\operatorname{Max}_{\mu}$, then $p$ is stable (see Remark 6.4) and defining

$$
d \rho=\frac{1}{|p(z, w)|^{2}} d \sigma
$$

we have that the inner products on $L^{2}(\mu)$ and $L^{2}(\rho)$ agree when restricted to $\square$. So, the moments agree:

$$
\int_{\mathbb{T}^{2}} z^{j} w^{k} d \mu=\int_{\mathbb{T}^{2}} z^{j} w^{k} d \rho \quad \text { for }|j| \leq n,|k| \leq m .
$$

Here is where we deviate from the Geronimo-Woerdeman proof. Observe that

$$
1=\int_{\mathbb{T}^{2}} \frac{|p(z, w)|}{\sqrt{t(z, w)}} \frac{\sqrt{t(z, w)}}{|p(z, w)|} \sigma \leq \sqrt{\int_{\mathbb{T}^{2}} \frac{|p(z, w)|^{2}}{t(z, w)} d \sigma} \sqrt{\int_{\mathbb{T}^{2}} \frac{t(z, w)}{|p(z, w)|^{2}} d \sigma}=\|p\|_{L^{2}(\mu) \sqrt{\|t\|_{L^{1}}(\rho)}}
$$

by Cauchy-Schwarz. Now, $\|p\|_{L^{2}(\mu)}=1$ since $p$ was chosen to have unit norm, and since the moments of $\mu$ and $\rho$ agree,

$$
\|t\|_{L^{1}(\rho)}=\|t\|_{L^{1}(\mu)}=\int_{\mathbb{T}^{2}} \frac{t(z, w)}{t(z, w)} d \sigma=1 .
$$

Therefore, we have equality in the above application of Cauchy-Schwarz (Equation (10-1)). So, $|p| / \sqrt{t}$ and $\sqrt{t} /|p|$ are multiples of one another, implying $|p|^{2}=c t$ for some constant $c$. The constant $c$ must be 1 since $p$ has unit norm in $L^{2}(\mu)$. Hence, $t(z, w)=|p(z, w)|^{2}$ for $(z, w) \in \mathbb{T}^{2}$.

We would like to extend this result to the case of nonnegative trigonometric polynomials, and we have some results in this direction. Work on characterizing when a nonnegative operator-valued two variable polynomial has a Fejér-Riesz type factorization was done in [Dritschel and Woerdeman 2005]. (Although the subtleties of all of the different candidates for the notion of outerness in several variables seem to have prevented getting a necessary and sufficient condition for a Fejér-Riesz factorization in that paper.)

We believe that any Fejér-Riesz type factorization for nonnegative two-variable trigonometric polynomials should take into account the notions of toral and atoral polynomials. These notions were alluded to in Remark 1.11 .

Example 10.2. Consider the nonnegative trigonometric polynomial $t(z, w)=|z-w|^{2}$. It cannot be factored as $|p(z, w)|^{2}$ where $p \in \mathbb{C}[z, w]$ has no zeros on the bidisk, because $p$ would necessarily vanish on the set $\left\{(z, w) \in \mathbb{T}^{2}: z=w\right\}$ and therefore $z-w$ would divide $p$. So, the polynomial $z w t(z, w)=2 z w-z^{2}-w^{2}$ associated to $t$ has a toral factor, and since this toral factor has zeros in the bidisk, there is no hope for such a Fejér-Riesz type of factorization. So, the question of whether a FejérRiesz factorization exists depends on the properties of the toral factors of $t$. This is true more generally.

Let $t: \mathbb{T}^{2} \rightarrow \mathbb{C}$ be a nonnegative trigonometric polynomial of two variables, given by

$$
t(z, w)=\sum_{j=-N}^{N} \sum_{k=-M}^{M} t_{j k} z^{j} w^{k} \geq 0,
$$

and let $q(z, w):=z^{N} w^{M} t(z, w) \in \mathbb{C}[z, w]$. 
Lemma 10.3. If $q$ has an irreducible toral factor $p$, then $p^{2}$ divides $q$, and $t /|p|^{2}$ is a nonnegative trigonometric polynomial.

Proof. Write $q=h p$ for some $h \in \mathbb{C}[z, w]$. By definition of toral, $p$ has infinitely many zeros on $\mathbb{T}^{2}$. The lemma is not difficult in the case where $p$ is a linear polynomial in one variable alone, so we assume this is not the case. Suppose $p$ has degree $(n, m)$. Let $\left(z_{0}, w_{0}\right) \in \mathbb{T}^{2} \cap Z_{p}$ with the property that $p\left(\cdot, w_{0}\right)$ has a zero of multiplicity one at $z_{0}$ and $t\left(\cdot, w_{0}\right)$ is not identically zero; this will be the case for all but finitely many of the $(z, w) \in \mathbb{T}^{2} \cap Z_{p}$. Now, $t\left(z, w_{0}\right)=z^{-N} w_{0}^{-M} h\left(z, w_{0}\right) p\left(z, w_{0}\right)$, and as $t\left(\cdot, w_{0}\right)$ is a nonnegative trig polynomial of one variable, it must have zeros of even order on $\mathbb{T}$. Hence, $h\left(z_{0}, w_{0}\right)=0$. Therefore, $h$ and $p$ share infinitely many zeros, and this implies $p$ divides $h$ by irreducibility of $p$. Hence, $p^{2}$ divides $q$. Toral polynomials are $\mathbb{T}^{2}$-symmetric in the sense that

$$
\overleftarrow{p}=c p
$$

for some unimodular constant $c$. So,

$$
t(z, w)=z^{-N} w^{-M} p(z, w)^{2} g(z, w)=z^{-N+n} w^{-M+m}|p(z, w)|^{2} g(z, w) \quad \text { for some } g \in \mathbb{C}[z, w] .
$$

Thus, $t /|p|^{2}$ is a nonnegative trig polynomial.

Corollary 10.4. If $t$ is a nonnegative trigonometric polynomial, then $t$ can be factored into $t(z, w)=$ $|p(z, w)|^{2} s(z, w)$ where $p \in \mathbb{C}[z, w]$ is a toral polynomial (or is a constant) and $s$ is a nonnegative trigonometric polynomial with finitely many zeros on $\mathbb{T}^{2}$.

The corollary divides the study of characterizing trig polynomials with a Fejér-Riesz factorization into the question of when a toral polynomial has no zeros on the bidisk and when a nonnegative trig polynomial finitely many zeros on the torus has a Fejér-Riesz factorization.

To introduce the next result we recall that every positive two variable trigonometric polynomial can be written as a sum of squares of two-variable polynomials. This was proved in [Dritschel 2004] and reproved in [Geronimo and Lai 2006] (the latter paper has a summary of related known results). It is unknown if all nonnegative trigonometric polynomials can be written as a sum of squares of two variable polynomials. The above corollary says that it is enough to address this question for trig polynomials with finitely many zeros. On the other hand, if it is true that all nonnegative trig polynomials are equal to a sum of squares of polynomials, then our approach allows us to characterize when they can be written as a single square of a polynomial with no zeros on the bidisk.

Theorem 10.5. Suppose $p_{1}, \ldots, p_{N} \in \mathbb{C}[z, w]$ have degree at most $(n, m)$ and no common factor. Also, assume that for some $j, p_{j}(0,0) \neq 0$. Let

$$
t(z, w)=\sum_{j=1}^{N}\left|p_{j}(z, w)\right|^{2} \text { for }(z, w) \in \mathbb{T}^{2},
$$

and define $d \mu=(1 / t) d \sigma$. The trigonometric polynomial $t$ can be written as $t(z, w)=|p(z, w)|^{2}$, where $p$ has no zeros on the bidisk, if and only if $\mu$ is an OC measure.

If every $p_{j}$ vanishes at the origin, we could apply a Möbius transformation to make sure not all of the polynomials vanish at the origin and then apply the above theorem to check whether the trig polynomial has the desired factorization. 
Proof. Our proof in the case of a strictly positive trig polynomial carries over with some modifications. The "only if" direction again follows from Theorem 7.4. Let us prove that if $\mu$ is an OC measure then $t$ has a Fejér-Riesz type of decomposition.

Since $t$ is of the given form it is clear that each $p_{j} \in L^{2}(\mu)$, as $\left|p_{j}\right|^{2} / t \leq 1$ on the torus. The assumption that $p_{j}(0,0) \neq 0$ guarantees that $\operatorname{Max}_{\mu}$ is nonempty (since we then know $\square_{\mu} \neq \square_{\mu}$ ). Let $q$ be a unit norm polynomial in $\operatorname{Max}_{\mu}$. By Corollary 6.5, $q$ is almost stable. To see this, note the corollary says $q$ can be factored as $q_{1} q_{2}$ where $q_{1}$ divides every element of $\square_{\mu}$ and $q_{2}$ is almost stable, but we assumed $p_{1}, \ldots, p_{N}$ have no common factor. Hence, $q_{1}$ must be a constant.

Define

$$
d \rho=\frac{1}{|q(z, w)|^{2}} d \sigma .
$$

By Corollary 7.6, $\square_{\mu}=\square_{\rho}$ and the inner products of $L^{2}(\mu)$ and $L^{2}(\rho)$ agree on $\square_{\mu}$. In particular,

$$
p_{j} / q \in L^{2}\left(\mathbb{T}^{2}\right) \quad \text { for each } j .
$$

Just as in the proof in the strictly positive case, we can prove

$$
1 \leq\|q\|_{L^{2}(\mu)} \sqrt{\|t\|_{L^{1}(\rho)}}
$$

by an application of Cauchy-Schwarz. Since $q$ has unit norm, $\|q\|_{L^{2}(\mu)}=1$, and since the inner products agree, we have

$$
\|t\|_{L^{1}(\rho)}=\sum_{j=1}^{N}\left\|p_{j}\right\|_{L^{2}(\rho)}^{2}=\sum_{j=1}^{N}\left\|p_{j}\right\|_{L^{2}(\mu)}^{2}=\|t\|_{L^{1}(\mu)}=1 .
$$

Therefore, just as in the proof for the strictly positive case, we have equality in Cauchy-Schwarz, which implies $t=|q|^{2}$ on the torus.

So, the above theorem addresses nonnegative trig polynomials of a specific form. The above proof would also work if we could decompose $t$ as

$$
t(z, w)=\sum_{j=1}^{N} p_{j}(z, w) \overline{q_{j}(z, w)}
$$

where $p_{j}, q_{j} \in L^{2}((1 / t) d \sigma)$ have no common factor and not all vanish at $(0,0)$.

Question 10.6. Can every nonnegative two variable trigonometric polynomial $t$ be decomposed as

$$
t(z, w)=\sum_{j=1}^{N} p_{j}(z, w) \overline{q_{j}(z, w)}
$$

where $p_{j}, q_{j}$ are in $L^{2}\left(\frac{1}{t} d \sigma\right)$ and have no common factor?

Next, we tackle toral factors of nonnegative trig polynomials.

Theorem 10.7. An irreducible toral polynomial $p \in \mathbb{C}[z, w]$ has no zeros in the bidisk if and only if

$$
\frac{\partial \overleftarrow{p}}{\partial z}+\frac{\overleftarrow{\partial p}}{\partial w}
$$


is almost stable. In this case, all of the zeros on $\mathbb{T}^{2}$ occur at singularities of $Z_{p}$ (i.e., common zeros of $\partial p / \partial z$ and $\partial p / \partial w)$.

The above reflections are performed at the degrees of $\partial p / \partial z$ and $\partial p / \partial w$ that would generically be expected. Namely, if $p$ has degree $(n, m)$, we reflect $\partial p / \partial z$ at the degree $(n-1, m)$.

Proof. If $p$ is toral, then $p$ is necessarily $\mathbb{T}^{2}$ symmetric, meaning $p$ is a unimodular constant times $\overleftarrow{p}$ (and in fact we may assume $p=\overleftarrow{p}$ by multiplying by an appropriate constant). It is proved in [Knese 2009] that if $p$ is $\mathbb{T}^{2}$ symmetric and has no zeros in the bidisk, then

$$
\frac{\partial p}{\partial z}+\frac{\check{\partial p}}{\partial w}
$$

has no zeros in the set $\overline{\mathbb{D}^{2}}$ except possibly at singularities of $Z_{p}$ (and there can be at most finitely many singularities).

Conversely, suppose $\partial p \overleftarrow{/} \partial z+\partial p \overleftarrow{/ \partial} w$ is almost stable. This implies

$$
\phi(z, w)=\frac{z(\partial p / \partial z)(z, w)+w(\partial p / \partial w)(z, w)}{(\partial p / \partial z)(z, w)+(\partial p / \partial w)(z, w)}
$$

is a (nonconstant) inner function on the bidisk, and must be bounded by 1 in modulus on the bidisk.

It is also proved in [Knese 2009] that if $p$ is $\mathbb{T}^{2}$ symmetric, then

$$
(n+m) p(z, w)=z \frac{\partial p}{\partial z}(z, w)+w \frac{\partial p}{\partial w}(z, w)+\frac{\partial p}{\partial z}(z, w)+\frac{\check{\partial p}}{\partial w}(z, w)
$$

So, if $p(z, w)=0$ for some $(z, w) \in \mathbb{D}^{2}$, then $|\phi(z, w)|=1$, which is a contradiction. Therefore, $p$ has no zeros in the bidisk.

Remark 10.8. We view this as progress on determining which nonnegative trig polynomials have a Fejér-Riesz decomposition for the following reasons. A nonnegative trig polynomial has a unique toral factor $|p|^{2}$ and determining whether $p$ has no zeros in the bidisk can be approached by looking at each factor of $p$. For the factors $f$ whose zero sets have no singularities on the torus, the above theorem says we can check whether $\partial \overleftarrow{f / \partial z}+\partial f \overleftarrow{f} \partial w$ is stable. A two-variable Schur-Cohn test, such as the one presented in [Geronimo and Woerdeman 2004], can be used to check this condition. For factors with singularities on the torus, one would need to adapt the Schur-Cohn test to the almost stable case. We leave this for future work.

To summarize, given a nonnegative trig polynomial $t$ we can factor it into $t(z, w)=|p(z, w)|^{2} s(z, w)$ where $p$ is a toral polynomial and $s$ is a nonnegative trig polynomial with finitely many zeros on $\mathbb{T}^{2}$. The above remark addresses cases where we can determine whether $p$ has no zeros in the bidisk. If $s$ has no zeros on the torus, the Geronimo-Woerdeman theorem characterizes whether it can be factored as $|q|^{2}$ where $q$ is stable. We have extended this characterization to a class of nonnegative trig polynomials with a special form, for which it is unknown whether this is all nonnegative trig polynomials. 


\section{Application: distinguished varieties}

One of our main applications is a bounded analytic extension theorem for distinguished varieties, which we now define.

Definition 11.1. A nonempty subset $V \subset \mathbb{C}^{2}$ is a distinguished variety if $V$ is an algebraic curve: there exists $p \in \mathbb{C}[z, w]$ such that

$$
V=\left\{(z, w) \in \mathbb{C}^{2}: p(z, w)=0\right\}
$$

and $V$ exits the bidisk through the distinguished boundary

$$
\partial\left(V \cap \overline{\mathbb{D}^{2}}\right) \subset \mathbb{T}^{2} .
$$

Our goal is to prove the following result. (This is a more qualitative version of Theorem 11.4 below.)

Theorem 11.2. Let $V \subset \mathbb{C}^{2}$ be a distinguished variety. Then, there is a rational function of $z, C(z)$, with no poles in $\mathbb{D}$, such that for every $f \in \mathbb{C}[z, w]$, there is a rational function $F \in \mathbb{C}(z, w)$, holomorphic on $\mathbb{D}^{2}$, which agrees with $f$ on $V$ :

$$
F(z, w)=f(z, w) \quad \text { for all }(z, w) \in V \cap \mathbb{D}^{2}
$$

and satisfies the estimate

$$
|F(z, w)| \leq|C(z)| \sup _{V \cap \mathbb{D}^{2}}|f| \quad \text { for all }(z, w) \in \mathbb{D}^{2} .
$$

If $V$ has no singularities on $\mathbb{T}^{2}, C(z)$ can be taken to be a constant.

The last statement is already proved in [Knese 2009]. Essentially, the purpose of this section is to inject the work of this paper into the work of [Knese 2009]. The use of the Cole-Wermer sums of squares formula is essential to the work in [Knese 2009], and if we use Theorem 1.3 in its place, the following lengthy theorem can be proved by slightly modifying the proofs in [Knese 2009].

Theorem 11.3. Let $V$ be a distinguished variety given as the zero set of a square-free polynomial $p \in$ $\mathbb{C}[z, w]$ of degree $(n, m)$. Let $a, b>0$ be positive real numbers. Then, there exist $\mathbf{P} \in \mathbb{C}^{n}[z, w], \operatorname{deg} \mathbf{P} \leq$ $(n-1, m)$, and $\mathbf{Q} \in \mathbb{C}^{m}[z, w], \operatorname{deg} \mathbf{Q} \leq(n, m-1)$ such that

- $\mathbf{P}$ is horizontally $\mathbb{D} \cup X_{2}$-stable and $\mathbf{Q}$ is vertically $\mathbb{D} \cup X_{1}$-stable where $X_{2}=\mathbb{T} \backslash \pi_{2}(S), X_{1}=\mathbb{T} \backslash \pi_{1}(S)$ and $S$ is the set of singularities of $V$;

- $(b m-a n)|p(z, w)|^{2}+2 \operatorname{Re}\left[\left(a z \frac{\partial p}{\partial z}(z, w)-b w \frac{\partial p}{\partial w}(z, w)\right) \overline{p(z, w)}\right]+\left(1-|z|^{2}\right)|\mathbf{P}(z, w)|^{2}$ $=\left(1-|w|^{2}\right)|\mathbf{Q}(z, w)|^{2} ;$

- there is a $m \times m$ matrix-valued rational inner function $\Phi: \mathbb{D} \rightarrow \mathbb{C}^{m \times m}$ such that $V$ has the following representation

$$
V \cap \mathbb{D}^{2}=\left\{(z, w) \in \mathbb{D}^{2}: \operatorname{det}\left(w I_{m}-\Phi(z)\right)=0\right\},
$$

and $\mathbf{Q}$ is a "polynomial eigenvector" for $\Phi$ :

$$
\Phi(z) \mathbf{Q}(z, w)=w \mathbf{Q}(z, w) \quad \text { for all }(z, w) \in V .
$$


Guide to the proof. Everything above is contained in a theorem in [Knese 2009] except for the horizontal and vertical stability of $\mathbf{P}, \mathbf{Q}$, respectively. So let us briefly outline how all of this can be done. All of the following are proved in [Knese 2009]:

(1) If $p \in \mathbb{C}[z, w]$ has degree $(n, m)$ and defines a distinguished variety, then the polynomial

$$
q(z, w)=z^{n} p\left(\frac{1}{z}, w\right)
$$

is $\mathbb{T}^{2}$-symmetric and has no zeros on the bidisk.

(2) Such a $q$ has the property that for each $a, b>0$

$$
a \frac{\grave{\partial q}}{\partial z}+b \frac{\overleftarrow{\partial q}}{\partial w}
$$

has no zeros on the closed bidisk $\overline{\mathbb{D}^{2}}$ except possibly at the finite number of singularities of $Z_{q}$, which necessarily occur on $\mathbb{T}^{2}$.

(3) Such a $q$ satisfies

$$
\begin{aligned}
(a n+b m)^{2}|q(z, w)|^{2}-2 \operatorname{Re}[( & \left.\left(a z q_{z}(z, w)+b w q_{w}(z, w)\right)(a n+b m) \overline{q(z, w)}\right] \\
& =\left|a \frac{\partial \underline{\partial q}}{\partial z}(z, w)+b \frac{\partial q}{\partial w}(z, w)\right|^{2}-\left|a z \frac{\partial q}{\partial z}(z, w)+b w \frac{\partial q}{\partial w}(z, w)\right|^{2}
\end{aligned}
$$

By Theorem 8.1, this last item (11-1) can written as

$$
\left(1-|z|^{2}\right)|\mathbf{E}(z, w)|^{2}+\left(1-|w|^{2}\right)|\mathbf{F}(z, w)|^{2}
$$

where $\mathbf{E}$ is horizontally $\mathbb{D} \cup Y_{2}$-stable and $\overleftarrow{\mathbf{F}}$ is vertically $\mathbb{D} \cup Y_{1}$-stable; here $Y_{2}=\mathbb{T} \backslash \pi_{2}\left(S_{q}\right), Y_{1}=\mathbb{T} \backslash \pi_{1}\left(S_{q}\right)$, and $S_{q}$ is the set of singularities of $q$. If we convert back to statements involving the polynomial $p$ (by replacing $z$ with $1 / z$ and multiplying by $z^{n}$ ) we get

$$
\begin{aligned}
(b m-a n)|p(z, w)|^{2}+2 \operatorname{Re}\left[\left(a z \frac{\partial p}{\partial z}(z, w)-b w \frac{\partial p}{\partial w}(z, w)\right) \overline{p(z, w)}\right]+\left(1-|z|^{2}\right)|\mathbf{P}(z, w)|^{2} \\
=\left(1-|w|^{2}\right)|\mathbf{Q}(z, w)|^{2},
\end{aligned}
$$

where $\mathbf{P}$ is horizontally $\mathbb{D} \cup X_{2}$-stable, $\mathbf{Q}$ is vertically $\mathbb{D} \cup X_{1}$-stable, $X_{2}=\mathbb{T} \backslash \pi_{2}(S), X_{1}=\mathbb{T} \backslash \pi_{1}(S)$, and $S$ is the set of singularities of $V$.

For the rest of the theorem, the proofs in [Knese 2009] can be applied unchanged.

Here is the promised bounded analytic extension theorem. The proof is identical to the proof in [Knese 2009] for distinguished varieties with no singularities on the torus. The only difference is that we did not have Theorem 1.3 to tell us that $\mathbf{Q}$ as above is vertically $\mathbb{D} \cup X_{1}$-stable, where $X_{1}=\mathbb{T} \backslash \pi_{1}(S)$. (In the case of no singularities we already knew $\mathbf{Q}$ is vertically $\overline{\mathbb{D}}$-stable.)

Let us write

$$
\mathbf{Q}(z, w)=Q(z) \Lambda_{m}(w),
$$

where the matrix polynomial $Q(z)$ is invertible for all $z \in \mathbb{D} \cup X_{1}$ (by definition of vertical $\mathbb{D} \cup X_{1}$ stability). 
Theorem 11.4. Let $V$ be a distinguished variety and let $\Phi, Q$, and $\mathbf{Q}$ be as in Theorem 11.3. Then, for any polynomial $f \in \mathbb{C}[z, w]$, the rational function

$$
F(z, w):=(1,0, \ldots, 0) Q(z)^{-1} f\left(z I_{m}, \Phi(z)\right) \mathbf{Q}(z, w)
$$

is equal to $f$ on $V \cap \mathbb{D}^{2}$ and we have the estimates

$$
|F(z, w)| \leq\left\|Q(z)^{-1}\right\||\mathbf{Q}(z, w)| \sup _{V \cap \mathbb{D}^{2}}|f| \leq \sqrt{m}\left\|Q(z)^{-1}\right\|\|Q(z)\| \sup _{V \cap \mathbb{D}^{2}}|f| \quad \text { for all }(z, w) \in \mathbb{D}^{2} .
$$

Here we are taking the operator norm of the matrices $Q(z)$ and $Q(z)^{-1}$.

In words, the growth of the extension $F$ is controlled by a rational function of one variable. When $V$ has no singularities on $\mathbb{T}^{2}, Q(z)$ is invertible for $z \in \overline{\mathbb{D}}$ and

$$
\sup _{\mathbb{D}}\left\|Q(z)^{-1}\right\|\|Q(z)\|
$$

is a finite constant.

The following is an example of the above two theorems.

Example 11.5. Consider the following reducible distinguished variety in $\mathbb{C}^{2}$

$$
V=\left\{(z, w) \in \mathbb{C}^{2}:(z-w)\left(z^{2}-w\right)=0\right\} .
$$

Like all distinguished varieties it has a determinantal representation of the following form:

$$
V \cap \mathbb{D}^{2}=\left\{(z, w) \in \mathbb{D}^{2}: \operatorname{det}(w I-\Phi(z))=0\right\},
$$

where $\Phi$ is a rational matrix valued inner function. One choice of $\Phi$ is

$$
\Phi(z)=\frac{1}{2}\left(\begin{array}{cc}
z(1+z) & z^{2}(1-z) \\
(1-z) & z(1+z)
\end{array}\right) .
$$

As can easily be checked

$$
\operatorname{det}\left(w I_{2}-\Phi(z)\right)=w^{2}-z w-z^{2} w+z^{3}=(w-z)\left(w-z^{2}\right) .
$$

The variety $V$ is simple yet instructive because it has a singularity at the origin and more importantly a singularity on the torus at the point $(1,1)$.

One choice for $\mathbf{Q}$ as above is

$$
\mathbf{Q}(z, w)=\left(\begin{array}{c}
2 w-z-z^{2} \\
1-z
\end{array}\right)
$$

Writing

$$
\mathbf{Q}(z, w)=Q(z)\left(\begin{array}{l}
1 \\
w
\end{array}\right)
$$

where

$$
Q(z)=\left(\begin{array}{cc}
-z-z^{2} & 2 \\
1-z & 0
\end{array}\right),
$$

we note that $Q(z)$ is invertible in $\overline{\mathbb{D}} \backslash\{1\}$; that is, $\mathbf{Q}$ is vertically $\overline{\mathbb{D}} \backslash\{1\}$-stable.

The analytic extension theorem now works as follows. 
Let $f \in \mathbb{C}[z, w]$ which we think of as a function on $V$. Then, the rational function

$$
F(z, w)=(1,0) Q(z)^{-1} f(z I, \Phi(z)) \mathbf{Q}(z, w)
$$

agrees with $f$ on $V$ because $\mathbf{Q}$ is a polynomial eigenvector for $\Phi$ on $V$. Furthermore, the size of $F$ on the bidisk can be estimated purely in terms of a fixed rational function of $z$ and the supremum of $f$ on $V \cap \mathbb{D}^{2}$.

Indeed,

$$
|F(z, w)| \leq\left|(1,0) Q(z)^{-1}\right||\mathbf{Q}(z, w)| \sup _{V \cap \mathbb{D}^{2}}|f| \leq \sqrt{1+\frac{16}{|1-z|^{2}}} \sup _{V \cap \mathbb{D}^{2}}|f| .
$$

\section{Application: Agler's Pick interpolation theorem}

As another application we give a simple proof of necessity in the Pick interpolation theorem on the bidisk. The proof below sidesteps the use of Andô's inequality and cone-separation arguments found in most proofs. (The proof of sufficiency can be accomplished with a "lurking isometry" argument; see Lemma 6.7 for something similar.) The proof is very similar to the argument in [Cole and Wermer 1999] for establishing Andô's inequality from the sum of squares decomposition.

Theorem 12.1 (Agler). Given distinct points

$$
\left(z_{1}, w_{1}\right), \ldots,\left(z_{N}, w_{N}\right) \in \mathbb{D}^{2}
$$

and complex numbers

$$
c_{1}, \ldots, c_{N} \in \mathbb{D},
$$

there exists a holomorphic function $f: \mathbb{D}^{2} \rightarrow \mathbb{D}$ which interpolates

$$
f\left(z_{j}, w_{j}\right)=c_{j} \text { for } j=1,2, \ldots, N
$$

if and only if there exist positive semidefinite $N \times N$ matrices $\Gamma$ and $\Delta$ such that

$$
1-c_{j} \overline{c_{k}}=\left(1-z_{j} \bar{z}_{k}\right) \Gamma_{j k}+\left(1-w_{j} \bar{w}_{k}\right) \Delta_{j k} .
$$

Proof of necessity. We first prove the theorem for rational inner functions and then use an approximation theorem to prove necessity in general. So, let $f$ be a rational inner function on the bidisk. Every rational inner function can be written as $f=\overleftarrow{p} / p$ for some $p \in \mathbb{C}[z, w]$ of degree at most $(n, m)$ having no zeros on the bidisk [Rudin 1969, Section 5.5.1]. Decomposing $p$ as in (8-1) and setting $(z, w)=\left(z_{j}, w_{j}\right)$ and $(Z, W)=\left(z_{k}, w_{k}\right)$ we have

$$
\begin{aligned}
p\left(z_{j}, w_{j}\right) \overline{p\left(z_{k}, w_{k}\right)}-\overleftarrow{p}\left(z_{j}, w_{j}\right) \overline{\overleftarrow{p}\left(z_{k}, w_{k}\right)} \\
=\left(1-z_{j} \overline{z_{k}}\right)\left\langle\mathbf{E}\left(z_{j}, w_{j}\right), \mathbf{E}\left(z_{k}, w_{k}\right)\right\rangle+\left(1-w_{j} \bar{w}_{k}\right)\left\langle\mathbf{F}\left(z_{j}, w_{j}\right), \mathbf{F}\left(z_{k}, w_{k}\right)\right\rangle
\end{aligned}
$$

Therefore, if $f\left(z_{j}, w_{j}\right)=(\overleftarrow{p} / p)\left(z_{j}, w_{j}\right)=c_{j}$, then

$$
\Gamma_{j k}=\frac{1}{p\left(z_{j}, w_{j}\right) \overline{p\left(z_{k}, w_{k}\right)}}\left\langle\mathbf{E}\left(z_{j}, w_{j}\right), \mathbf{E}\left(z_{k}, w_{k}\right)\right\rangle, \quad \Delta_{j k}=\frac{1}{p\left(z_{j}, w_{j}\right) \overline{p\left(z_{k}, w_{k}\right)}}\left\langle\mathbf{F}\left(z_{j}, w_{j}\right), \mathbf{F}\left(z_{k}, w_{k}\right)\right\rangle
$$


are both positive semidefinite matrices and they satisfy

$$
1-c_{j} \overline{c_{k}}=\left(1-z_{j} \overline{z_{k}}\right) \Gamma_{j k}+\left(1-w_{j} \bar{w}_{k}\right) \Delta_{j k},
$$

as desired.

In general, suppose $f: \mathbb{D}^{2} \rightarrow \mathbb{D}$ is holomorphic and $f\left(z_{j}, w_{j}\right)=c_{j}$. Rudin's extension of Carathéodory's theorem to the polydisk [Rudin 1969, Theorem 5.5.1] says that $f$ is the pointwise limit of a sequence of rational inner functions: $f_{\alpha} \rightarrow f$ as $\alpha \rightarrow \infty$, where $\alpha$ is used to index the positive integers. Corresponding to each such rational inner function $f_{\alpha}$, we write $f_{\alpha}\left(z_{j}, w_{j}\right)=c_{\alpha, j}$ and we choose positive semidefinite matrices $\Gamma_{\alpha}, \Delta_{\alpha}$ so that an equation analogous to (12-1) holds:

$$
1-c_{\alpha, j} \bar{c}_{\alpha, k}=\left(1-z_{j} \bar{z}_{k}\right)\left(\Gamma_{\alpha}\right)_{j k}+\left(1-w_{j} \bar{w}_{k}\right)\left(\Delta_{\alpha}\right)_{j k} .
$$

The set of positive semidefinite matrices (of a fixed size) with diagonal entries bounded by some constant is compact (their operator norms are bounded by their traces which are uniformly bounded). The diagonal entries of $\Gamma_{\alpha}$ and $\Delta_{\alpha}$ are bounded independently of $\alpha$ (e.g., it is not hard to prove

$$
\frac{1}{1-\left|z_{j}\right|^{2}} \geq\left(\Gamma_{\alpha}\right)_{j j}
$$

for $j=1, \ldots, N)$ and therefore we may choose a subsequence so that $\Gamma_{\alpha}$ converges to some positive semidefinite matrix $\Gamma$ and $\Delta_{\alpha}$ converges to some positive semidefinite matrix $\Delta$. Therefore, if we take the limit as $\alpha \rightarrow \infty$ in Equation (12-2) we have proved

$$
1-c_{j} \overline{c_{k}}=\left(1-z_{j} \overline{z_{k}}\right) \Gamma_{j k}+\left(1-w_{j} \bar{w}_{k}\right) \Delta_{j k},
$$

which proves necessity in general.

Question 12.2. Can the uniqueness in Theorem 1.3 be carried over in some way to the above theorem?

Solutions to extremal Pick problems in two variables (those solvable with a function of norm one but no less) are not unique as they are in one variable, so we are necessarily vague in our question.

\section{Questions}

We have already asked three questions: Questions 5.8, 10.6, and 12.2. Here are two others. One of the most fundamental questions to come out of our research is the following:

Question 13.1. When is a rational function $p / q$ in $L^{2}\left(\mathbb{T}^{2}\right)$ ?

Here we may as well assume $p, q \in \mathbb{C}[z, w]$ are relatively prime but we are otherwise not imposing any conditions on their zero sets. If we impose restrictions, we can ask a more concrete question.

Suppose $q \in \mathbb{C}[z, w]$ is almost stable, $\operatorname{deg} q=(n, m)$, and suppose $p \in \mathbb{C}[z, w]$ has degree $\leq$ $(n-1, m-1)$. If $p / q \in L^{2}\left(\mathbb{T}^{2}\right)$, then the sums of squares decomposition (as in Theorem 6.1) tells us that there is a constant $c$ such that

$$
|q(z, w)|^{2}-|\overleftarrow{q}(z, w)|^{2} \geq c\left(1-|z|^{2}\right)\left(1-|w|^{2}\right)|p(z, w)|^{2} \quad \text { for }(z, w) \in \mathbb{D}^{2}
$$

since $p$ will be in $\mathbf{\square}_{\mu}$ for the Bernstein-Szegő measure $\mu$ associated to $q$.

Question 13.2. Is the converse true? Does the estimate (13-1) imply $p / q \in L^{2}\left(\mathbb{T}^{2}\right)$ ? 


\section{Notational index and conventions}

In this section we index where various notations and terms are defined in the paper. We also list our notational conventions.

stable/almost stable vertically stable

$\Lambda_{n}(z), \Lambda_{m}(w)$

atoral

distinguished variety

degree $(n, m)$

$\square, \square, \square, \square, \square, \square$

$K V, K \square \square_{\mu}$, etc.

$\operatorname{Max}_{\mu}, \operatorname{Min}_{\mu}$

OC measure

$L_{(Z, W)}$

$Z_{q}$
Definition 1.4

Definition 1.6

Equation (1-3)

Definition 1.13

Definition 11.1

Definition 1.8

Notation 4.3

Notation 4.1

Equations (4-7) and (4-8)

Definition 6.2

Equation (7-1)

Equation (7-5)

horizontally stable
reflection $\overleftarrow{q}(z, w)$
toral
divisor of ideal
$d \sigma=d \sigma(z, w)$
$\hat{q}(j, k)$
$\langle f, g\rangle_{\mu}$
$w \boldsymbol{\square}_{\mu}, z \mathbf{\square}_{\mu}, \mathbf{\Xi}_{\mu}, \Xi_{\mu}$, etc.
$\Phi_{\mu}$
$\mathbb{T}^{2}$-symmetric
$\pi_{1}, \pi_{2}$
$\mathbb{C}^{N}\left[z, \mathbb{C}^{N}[z, w], \mathbb{E}\right.$

Definition 1.6

Definition 1.9

Definition 1.12

Definition 5.1

Equation (4-1)

Equation (4-4)

Equation (4-3)

Notation 4.4

Equation (4-2)

Definition 5.6

Equation (7-6)

Notation 1.5

$$
\begin{aligned}
& n, m \\
& p, q \\
& \mathbf{E}, \mathbf{F}, \mathbf{G}, \mathbf{A}, \mathbf{B}, \mathbf{Q} \\
& E, F, A, B, Q \\
& \langle\cdot, \cdot\rangle \text { with no subscript } \\
& L^{2}\left(\mathbb{T}^{2}\right) \\
& L^{2}(\mu), L^{2}(\rho) \\
& H^{2}(\mathbb{T}), H^{2}\left(\mathbb{T}^{2}\right) \\
& \Phi, \Psi
\end{aligned}
$$

fixed positive integers (see Remark 4.2)

elements of $\mathbb{C}[z, w]$

vector polynomials

matrix polynomials in one variable inner product on $\mathbb{C}^{N}$ ( $N$ determined from context) $L^{2}$ on the torus with respect to Lebesgue measure $L^{2}$ on the torus with respect to the measure $\mu$ or $\rho$ classical Hardy space on $\mathbb{T}$ or $\mathbb{T}^{2}$ one variable matrix valued inner functions

\section{Acknowledgements}

The author would like to sincerely thank John McCarthy and the anonymous referee for sharing their thoughts on this paper.

\section{References}

[Agler et al. 2006] J. Agler, J. E. McCarthy, and M. Stankus, “Toral algebraic sets and function theory on polydisks", J. Geom. Anal. 16:4 (2006), 551-562. MR 2007j:32002 Zbl 1103.14019

[Agler et al. 2008] J. Agler, J. E. McCarthy, and M. Stankus, "Local geometry of zero sets of holomorphic functions near the torus", New York J. Math. 14 (2008), 517-538. MR 2010a:32014 Zbl 1153.14002

[Cole and Wermer 1999] B. J. Cole and J. Wermer, "Ando's theorem and sums of squares", Indiana Univ. Math. J. 48:3 (1999), 767-791. MR 2000m:47014 Zbl 0945.47010

[D'Angelo 1993] J. P. D'Angelo, Several complex variables and the geometry of real hypersurfaces, Studies in Advanced Mathematics, CRC Press, Boca Raton, FL, 1993. MR 94i:32022 Zbl 0854.32001

[Dritschel 2004] M. A. Dritschel, "On factorization of trigonometric polynomials", Integral Equations Operator Theory 49:1 (2004), 11-42. MR 2005d:47034 Zbl 1070.47011 
[Dritschel and Woerdeman 2005] M. A. Dritschel and H. J. Woerdeman, "Outer factorizations in one and several variables", Trans. Amer. Math. Soc. 357:11 (2005), 4661-4679. MR 2006e:47039 Zbl 1073.47024

[Duren 1970] P. L. Duren, Theory of $H^{p}$ spaces, Pure and Applied Mathematics 38, Academic Press, New York, 1970. MR 42 \#3552 Zbl 0215.20203

[Geronimo and Lai 2006] J. S. Geronimo and M.-J. Lai, "Factorization of multivariate positive Laurent polynomials", J. Approx. Theory 139:1-2 (2006), 327-345. MR 2007a:47023 Zbl 1099.12001

[Geronimo and Woerdeman 2004] J. S. Geronimo and H. J. Woerdeman, "Positive extensions, Fejér-Riesz factorization and autoregressive filters in two variables", Ann. of Math. (2) 160:3 (2004), 839-906. MR 2006b:42036

[Knese 2008] G. Knese, "Bernstein-Szegó measures on the two dimensional torus", Indiana Univ. Math. J. 57:3 (2008), 13531376. MR 2009h:46054

[Knese 2009] G. Knese, "Polynomials defining distinguished varieties", preprint, 2009. To appear in Trans. Amer. Math. Soc. arXiv 0909.1818v1

[Rudin 1969] W. Rudin, Function theory in polydiscs, W. A. Benjamin, New York, 1969. MR 41 \#501 Zbl 0177.34101

[Simon 2005] B. Simon, Orthogonal polynomials on the unit circle. Part 1, American Mathematical Society Colloquium Publications 54, American Mathematical Society, Providence, RI, 2005. MR 2006a:42002a Zbl 1082.42020

[Simon 2008] B. Simon, "The Christoffel-Darboux kernel”, pp. 295-335 in Perspectives in partial differential equations, harmonic analysis and applications, Proc. Sympos. Pure Math. 79, Amer. Math. Soc., Providence, RI, 2008. MR 2010d:42045 Zbl 1159.42020

Received 23 Oct 2008. Revised 20 Oct 2009. Accepted 3 Dec 2009.

GREG KNESE: gknese@uci.edu

University of California, Irvine, Department of Mathematics, Irvine CA 92697-3875, United States

http://www.math.uci.edu/ gknese 


\title{
Analysis \& PDE
}

pjm.math.berkeley.edu/apde

EDITORS

\author{
EDITOR-IN-CHIEF \\ Maciej Zworski \\ University of California \\ Berkeley, USA
}

BOARD OF EDITORS

\begin{tabular}{|c|c|c|c|}
\hline Michael Aizenman & $\begin{array}{l}\text { Princeton University, USA } \\
\text { aizenman@math.princeton.edu }\end{array}$ & Nicolas Burq & $\begin{array}{l}\text { Université Paris-Sud 11, France } \\
\text { nicolas.burq @math.u-psud.fr }\end{array}$ \\
\hline Luis A. Caffarelli & $\begin{array}{l}\text { University of Texas, USA } \\
\text { caffarel@math.utexas.edu }\end{array}$ & un-Yung Alice Chang & $\begin{array}{l}\text { Princeton University, USA } \\
\text { chang@math.princeton.edu }\end{array}$ \\
\hline Michael Christ & $\begin{array}{l}\text { University of California, Berkeley, USA } \\
\text { mchrist@math.berkeley.edu }\end{array}$ & Charles Fefferman & $\begin{array}{l}\text { Princeton University, USA } \\
\text { cf@math.princeton.edu }\end{array}$ \\
\hline Ursula Hamenstaedt & $\begin{array}{l}\text { Universität Bonn, Germany } \\
\text { ursula@math.uni-bonn.de }\end{array}$ & Nigel Higson & $\begin{array}{l}\text { Pennsylvania State Univesity, USA } \\
\text { higson@ math.psu.edu }\end{array}$ \\
\hline Vaughan Jones & $\begin{array}{l}\text { University of California, Berkeley, USA } \\
\text { vfr@math.berkeley.edu }\end{array}$ & Herbert Koch & $\begin{array}{l}\text { Universität Bonn, Germany } \\
\text { koch@math.uni-bonn.de }\end{array}$ \\
\hline Izabella Laba & $\begin{array}{l}\text { University of British Columbia, Canada } \\
\text { ilaba@math.ubc.ca }\end{array}$ & Gilles Lebeau & $\begin{array}{l}\text { Université de Nice Sophia Antipolis, France } \\
\text { lebeau@unice.fr }\end{array}$ \\
\hline László Lempert & $\begin{array}{l}\text { Purdue University, USA } \\
\text { lempert@math.purdue.edu }\end{array}$ & Richard B. Melrose & $\begin{array}{l}\text { Massachussets Institute of Technology, USA } \\
\text { rbm@math.mit.edu }\end{array}$ \\
\hline Frank Merle & $\begin{array}{l}\text { Université de Cergy-Pontoise, France } \\
\text { Frank.Merle@u-cergy.fr }\end{array}$ & William Minicozzi II & $\begin{array}{l}\text { Johns Hopkins University, USA } \\
\text { minicozz@ math.jhu.edu }\end{array}$ \\
\hline Werner Müller & $\begin{array}{l}\text { Universität Bonn, Germany } \\
\text { mueller@math.uni-bonn.de }\end{array}$ & Yuval Peres & $\begin{array}{l}\text { University of California, Berkeley, USA } \\
\text { peres@stat.berkeley.edu }\end{array}$ \\
\hline Gilles Pisier & $\begin{array}{l}\text { Texas A\&M University, and Paris } 6 \\
\text { pisier@math.tamu.edu }\end{array}$ & Tristan Rivière & $\begin{array}{l}\text { ETH, Switzerland } \\
\text { riviere@math.ethz.ch }\end{array}$ \\
\hline Igor Rodnianski & $\begin{array}{l}\text { Princeton University, USA } \\
\text { irod@ math.princeton.edu }\end{array}$ & Wilhelm Schlag & $\begin{array}{l}\text { University of Chicago, USA } \\
\text { schlag@math.uchicago.edu }\end{array}$ \\
\hline Sylvia Serfaty & $\begin{array}{l}\text { New York University, USA } \\
\text { serfaty@ cims.nyu.edu }\end{array}$ & Yum-Tong Siu & $\begin{array}{l}\text { Harvard University, USA } \\
\text { siu@math.harvard.edu }\end{array}$ \\
\hline Terence Tao & $\begin{array}{l}\text { University of California, Los Angeles, USA } \\
\text { tao@math.ucla.edu }\end{array}$ & A Michael E. Taylor & $\begin{array}{l}\text { Univ. of North Carolina, Chapel Hill, USA } \\
\text { met@math.unc.edu }\end{array}$ \\
\hline Gunther Uhlmann & $\begin{array}{l}\text { University of Washington, USA } \\
\text { gunther@math.washington.edu }\end{array}$ & András Vasy & $\begin{array}{l}\text { Stanford University, USA } \\
\text { andras@math.stanford.edu }\end{array}$ \\
\hline Virgil Voiculescu & $\begin{array}{l}\text { University of California, Berkeley, USA } \\
\text { dvv@math.berkeley.edu }\end{array}$ & Steven Zelditch & $\begin{array}{l}\text { Johns Hopkins University, USA } \\
\text { szelditch@math.jhu.edu }\end{array}$ \\
\hline
\end{tabular}

\section{PRODUCTION}

apde@mathscipub.org

Paulo Ney de Souza, Production Manager Sheila Newbery, Production Edito

Silvio Levy, Senior Production Editor

See inside back cover or pjm.math.berkeley.edu/apde for submission instructions.

The subscription price for 2010 is US \$120/year for the electronic version, and \$180/year for print and electronic. Subscriptions, requests for back issues from the last three years and changes of subscribers address should be sent to Mathematical Sciences Publishers, Department of Mathematics, University of California, Berkeley, CA 94720-3840, USA.

Analysis \& PDE, at Mathematical Sciences Publishers, Department of Mathematics, University of California, Berkeley, CA 94720-3840 is published continuously online. Periodical rate postage paid at Berkeley, CA 94704, and additional mailing offices.

APDE peer-review and production is managed by EditFLOW ${ }^{\mathrm{TM}}$ from Mathematical Sciences Publishers.

PUBLISHED BY

mathematical sciences publishers

http://www.mathscipub.org

A NON-PROFIT CORPORATION

Typeset in IATEX $_{\mathrm{E}}$

Copyright @2010 by Mathematical Sciences Publishers 


\section{ANALYSIS \& PDE}

\section{Volume $3 \quad$ No. $2 \quad 2010$}

Polynomials with no zeros on the bidisk

\section{GREG KNESE}

Local wellposedness for the 2+1-dimensional monopole equation Magdalena CZubaK

Regularity of almost periodic modulo scaling solutions for mass-critical NLS and applications DONG LI and XIAOYI ZHANG

Estimées des noyaux de Green et de la chaleur sur les espaces symétriques GILLES CARRON

Lower bounds for resonances of infinite-area Riemann surfaces 